\title{
Vortex Sound Models: Passive and Active Noise Control
}

\author{
Matthew J. Priddin *, David I. Baker ${ }^{\dagger}$, Lorna J. Ayton ${ }^{\star}$ and Nigel Peake ${ }^{\S}$ \\ University of Cambridge, Cambridge, UK CB3 OWA
}

\begin{abstract}
Simple models of vortex sound, considering the motion of a line vortex past an object, have been used to determine the far-field noise due to an idealised hydrodynamic disturbance, modelling, for example, instabilities within a turbulent boundary layer. Extensions of such models to investigate devices designed for control of trailing-edge noise, both passively and actively, are proposed. This simple mathematical framework potentially gives insight into the mechanism behind experimentally observed reductions in far-field noise.
\end{abstract}

\section{Introduction}

Airframe noise is often the dominant contribution to the sound heard from an aircraft, for example on approach. Over the past few decades certification requirements have gradually tightened, reaching the point where the required noise reductions cannot be achieved through reducing engine noise alone [1]. There are, similarly, restrictions on the noise output of wind turbines so as to reduce their impacts on local communities and wildlife. In practice this is achieved by braking the turbines at high speed, reducing energy outputs and efficiency. The mechanism of noise generation from aircraft wings and turbine blades is comparable, in particular the contribution of the sharp trailing-edge of the aerofoil. This trailing-edge is a well understood and unavoidable source of noise. Vortical disturbances within a turbulent boundary layer interact with the sharp trailing-edge of an aerofoil, with the resulting scattered pressure fluctuations propagating to the far-field as sound. The sound intensity of this noise source scales with velocity as $U^{3} M^{2}$ independently of the behaviour of the unsteady flow [2], with $M=U / c_{0}$ the flow Mach number. This should be compared to the classical result of sound intensity of turbulence with no hard boundaries within the flow, scaling as $U^{3} M^{5}[3]$.

Since this sharp trailing-edge is necessary aerodynamically for lift, controlling this noise is difficult, and a variety of noise-control devices have been proposed which modify the airflow around the tip, both passive and active. Taking inspiration from the silent flight of owls, Clark et al. [4-6] developed finlets, streamwise-oriented fence-like objects near the trailing-edge, a development from fibres near the trailing-edge on the wings of many species of owl. Similar objects have been investigated experimentally and numerically [7--9], and objects show a reduction in far-field noise due to trailing-edge scattering. Other passive noise-control devices include serrated trailing-edges, both sinusoidal and sawtooth [10, 11] . The trailing-edge reduction observed here is related to the three-dimensional nature of the trailing-edge geometry, and is not directly considered in this paper.

Active flow control offers additional methods of flow manipulation, and a distinct advantage over passive mechanisms in the ability to adapt to the external flow environment. This versatility is clearly applicable to current noise reduction objectives. For instance, aircraft take-off and landing scenarios, and the variety of atmospheric conditions faced by wind turbines. Adaptability also narrows the domain in which any particular implementation must be effective. Recent numerical and experimental studies [12, 13] have indicated that active blowing or suction could achieve an appreciable reduction of trailing edge noise for an acceptable energy expense. Szóke and Azarpeyvand [13] experimentally investigated blowing and suction for a nominally $2 \mathrm{D}$ flow over a sharp trailing edge, finding a reduction of up to $10-15 \mathrm{~dB}$ in surface pressure fluctuations. The associated far-field sound reduction may then be inferred from Amiet's model [14]. The mechanism responsible for the pressure fluctuation reduction is believed to be the manipulation of the boundary layer flow, controlling the location and intensity of turbulent structures within the boundary layer.

Theoretical investigations into active flow face the difficulty of accurately modelling the control device in addition to any underlying complexity of the flow considered. Boundary layer manipulation by active control has been been extensively studied with view to reducing drag, seeking to delay transition to turbulence or to achieve relaminarization [15-17] theoretical work on active control for acoustic applications have focussed on sound cancellation: using actuators to generate anti-noise that destructively interferes with an incident disturbance, or acoustic feedback [18, 19]. Few

\footnotetext{
*Graduate Student, Department of Applied Mathematics and Theoretical Physics, Student Member AIAA.

Graduate Student, Department of Applied Mathematics and Theoretical Physics, Student Member AIAA.

\#PSRC Early Career Fellow, Churchill College and the Department of Applied Mathematics and Theoretical Physics.

${ }^{\S}$ Professor, Department of Applied Mathematics and Theoretical Physics, Senior Member AIAA.
} 
theoretical investigations into the acoustic consequences of flow manipulation by active control exist. Simple models of active flow control methods currently considered may be constructed in vortex sound framework, which we demonstrate here. Despite neglecting many details of boundary layer trailing edge active device interactions required for a full understanding, such models may offer valuable insights by demonstrating the role of well-defined physical processes, so elucidating the significance of additional modelling complexities. More generally, rapidly computable models are particularly relevant to optimization and control problems on high-dimensional parameter spaces. If sufficiently realistic, these models could provide a useful guide to more costly numerical simulations and experimental work.

The consideration of the idealised model of the motion of a single line vortex passing an object, in irrotational flow, was used to derive the $U^{5}$ scaling law for trailing-edge noise efficiently [20]. It has been extended to consider general aerofoils and thick trailing-edges [21-23], and provides a straightforward analytical model for direct prediction of far-field sound from vortical motion in the flow, as a simplified model for turbulence. Recent developments have included the addition of flexible fibres to trailing edge [24] and fence-like structures to a thick aerofoil [25, 26], as a qualitative model for the effect of finlets or other similar passive noise control devices [24]. The process typically involves some sort of spanwise average of the device to reduce the problem to a quasi-two dimensional one, onto which we can apply conformal mapping theory to obtain the results of interest. Such models, and similar ones for active control, are elucidated in this work, qualitatively suggesting some component of noise reduction due to the movement of turbulence from the trailing-edge.

This paper begins with an overview of the theory of vortex sound in \$I with specific application to the movement of a line vortex past a trailing-edge (which may have $O(1)$ geometry, though we assume it is thin on the acoustic lengthscales of interest). The following section, $\$$ III] models fence-like passive noise-control devices through a spanwise average reducing the problem to a nearly two-dimensional one. Direct inclusion of blowing and suction near a trailing-edge are then considered in $\$[\mathrm{IV}$, with the dependence on the location and strength of the control device, with respect to the trailing-edge and uniform flow strength, investigated in detail. The conclusions that can be drawn from these models (and those that can't) are summarised in $\$ \mathrm{~V}$.

\section{Vortex sound and motion in two dimensions}

\section{A. Vortex motion}

\section{Vortex motion in general potential flow}

In a general, incompressible flow, vorticity $\omega=\nabla \times \mathbf{u}$ satisfies the vorticity equation

$$
\frac{D \omega}{D t}=(\omega \cdot \nabla) \mathbf{u}+v \nabla^{2} \omega .
$$

If we consider the limit of inviscid, two-dimensional flow, so that $\omega=\omega \mathbf{e}_{3}$ and viscosity $v$ can be neglected (the limit of high-Reynolds number), then this equation simplifies to

$$
\frac{D \omega}{D t}=0,
$$

that is, vorticity is convected with the mean flow. The material derivative is here defined as

$$
\frac{D}{D t}=\frac{\partial}{\partial t}+\mathbf{u} \cdot \nabla
$$

It is possible to compute the trajectory of a line vortex, confined to a point, in otherwise irrotational background flow, including the effects of self-action. For sufficiently simple geometries, this problem becomes one of integrating a straightforward ODE, determined by the identification of suitable conformal maps. In complex notation, with $z=x_{1}+i x_{2}$ and $W=\phi+i \psi$ the complex potential of the total flow, the combination of the steady background flow and the vortex-induced flow, the vortex (of strength $\Gamma$ ) has position $z_{0}$ satisfying

$$
\frac{\mathrm{d} z_{0}^{*}}{\mathrm{~d} t}=\frac{\mathrm{d}}{\mathrm{d} z}\left(W-\frac{\Gamma}{2 \pi \mathrm{i}} \log \left(z-z_{0}\right)\right)_{z=z_{0}} .
$$

From the complex potential $W$, the horizontal and vertical components of the flow field are computed via

$$
u-\mathrm{i} v=\frac{\mathrm{d} W}{\mathrm{~d} z}
$$


and we see that $z_{0}$ moves with the flow (once the singularity at the vortex itself is removed). Provided a conformal map $\zeta(z)$ can be found mapping the geometry of interest to the upper-half complex $\zeta$-plane, shown in Figure 1 it is easy to synthesise the background flow, the self-induced vortex motion and the far-field acoustic Green's function (see the next section) as straightforward functions of $\zeta$.

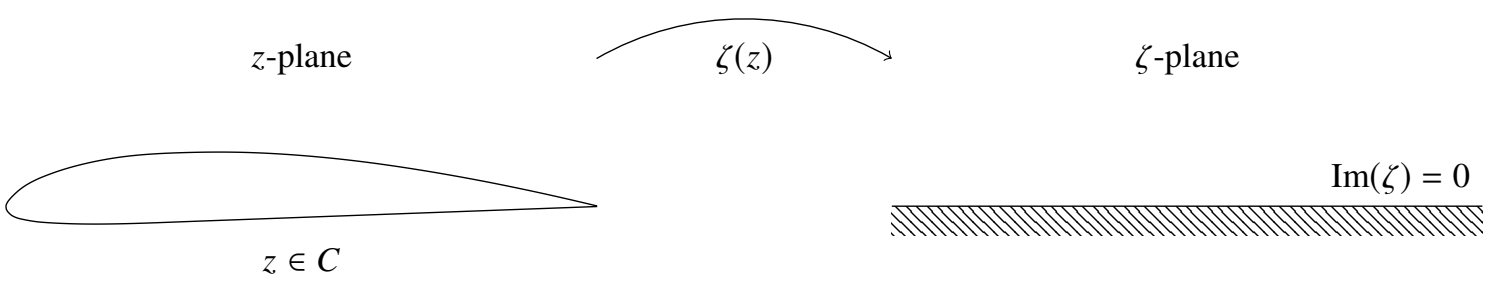

Fig. 1 Consider $\zeta(z)$ a complex analytic function of $z=x_{1}+\mathrm{i} x_{2}$, such that the boundary of our domain (e.g. the interior boundary $z \in C$ shown on the left), with $\operatorname{Im}(\zeta(z))=0$ for $z \in C$. This simplifies the geometry of the problem considerably, and and exact solutions to Laplace's equation can be found using the resulting map, allowing incompressible, irrotational and inviscid flows to be determined from $\zeta$ alone.

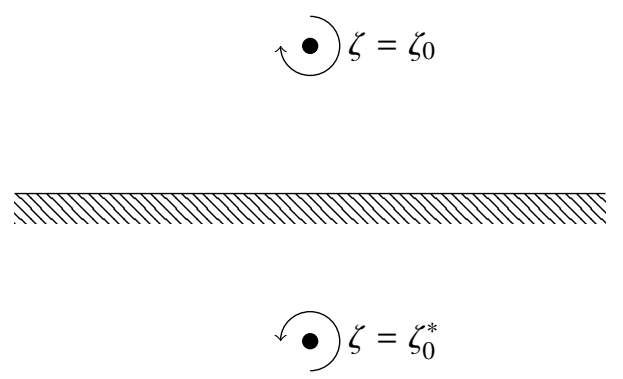

Fig. 2 The flow field induced by a vortex (of strength $\Gamma$, shown as positive above) at $\zeta$-location $\zeta_{0}$ can be obtained simply by the addition of an image vortex at the conjugate point $\zeta_{0}^{*}$ with opposite strength $-\Gamma$.

In the $\zeta$-plane, the solution for the vortex-induced flow field, with vortex $\zeta$-location $\zeta_{0}=\zeta\left(z_{0}\right)$, can be written down exactly (using the method of images, see Figure 2), with complex potential

$$
W_{v}=\frac{\Gamma}{2 \pi \mathrm{i}}\left(\log \left(\zeta-\zeta_{0}\right)-\log \left(\zeta-\zeta_{0}^{*}\right)\right)
$$

If we decompose $W=W_{b}+W_{v}$, where $W_{b}$ is the complex velocity potential for whatever inviscid, irrotational background flow we choose to impose, then we have

$$
\frac{\mathrm{d} z_{0}^{*}}{\mathrm{~d} t}=\left.\frac{\mathrm{d} W_{b}}{\mathrm{~d} z}\right|_{z_{0}}+\frac{\mathrm{d}}{\mathrm{d} z}\left(W_{v}-\frac{\Gamma}{2 \pi \mathrm{i}} \log \left(z-z_{0}\right)\right)_{z=z_{0}} .
$$

We can explicitly evaluate the final term, with the log-like singularity in (6) cancelling to give

$$
\frac{\mathrm{d} z_{0}^{*}}{\mathrm{~d} t}=\left.\frac{\mathrm{d} W_{b}}{\mathrm{~d} z}\right|_{z_{0}}+\frac{\Gamma}{2 \pi \mathrm{i}}\left[\frac{\zeta_{0}^{\prime \prime}}{2 \zeta_{0}^{\prime}}-\frac{\zeta_{0}^{\prime}}{\zeta_{0}-\zeta_{0}^{*}}\right]
$$

Equivalently vortex motion in the $\zeta$-plane can be considered, which is more convenient if the inverse map $z(\zeta)$ is more readily available, mapping the real axis $\operatorname{Im}(\zeta)=0$ to the boundary of the object of interest, with projected vortex $\zeta$-position evolving as

$$
\frac{\mathrm{d} \zeta_{0}^{*}}{\mathrm{~d} t}=\left.\frac{1}{\left|z_{0}^{\prime}\right|^{2}} \frac{\mathrm{d} W_{b}}{\mathrm{~d} \zeta}\right|_{\zeta_{0}}-\frac{1}{\left|z_{0}^{\prime}\right|^{2}} \frac{\Gamma}{2 \pi \mathrm{i}}\left[\frac{z_{0}^{\prime \prime}}{2 z_{0}^{\prime}}+\frac{1}{\zeta_{0}-\zeta_{0}^{*}}\right]
$$

with subscript 0 denoting evaluation at $\zeta_{0}$. 


\section{Vortex motion past a trailing-edge}

Whilst, in principle, these results can be applied to arbitrary domains, the remainder of this discussion will focus on trailing-edges. Explicitly, we shall look at bodies with interior bounded between $-c_{1}<x_{2}<c_{2}$ as $x_{1} \rightarrow-\infty$, with $c_{1,2} \geq 0$. The most trivial case is the semi-infinite flat plate, with the exterior domain $z=x_{1}+\mathrm{i} x_{2}$ mapped to the Upper Half $\zeta$-Plane (UH $\zeta$ P) via the map

$$
\zeta=i \sqrt{z}
$$

with the principal branch cut taken (along the negative real axis). This transform enforces a normalisation on the all other trailng-edge transforms: for $\zeta_{X}$ mapping exterior domain $X$ to the $\mathrm{UH} \zeta \mathrm{P}$, we impose the normalisation

$$
\zeta_{X}(z) \sim i \sqrt{z} \text { as } z \rightarrow \infty
$$

alongside the usual condition that $\zeta_{X}$ maps the boundary of $X, \partial X$, to the real $\zeta$-axis. This normalisation has the benefit that writing down both the inviscid flow that is uniform around the plate, and (as shall be demonstrated later) the compact Green's function, are straightforward functions of $\zeta$.

Uniform flow downstream. We firstly suppose that in the $z$-plane, the real geometry, that the background complex potential $W_{b} \sim U z$ for $z$ large. This corresponds to a flow that is uniform and in the $x_{1}$ direction (a condition forced by the blocked domain stretching to $x_{1}=-\infty$ ). Since $z \sim-\zeta^{2}$, a solution to the irrotational flow problem, perturbed by the trailing-edge geometry, is given in the $\zeta$-plane simply by $-U \zeta^{2}$. This satisfies the no-penetration condition on the real $\zeta$-axis (equivalent to $\operatorname{Im}\left(W_{b}\right)$ being constant on $\operatorname{Im}(\zeta)=0$ - the surface of the body is a streamline). This is not quite unique, the solution

$$
W_{b}=-U\left(\zeta-\zeta_{s}\right)^{2}
$$

is also a solution for constant real $\zeta_{s}$. This choice of stagnation point for the flow must be chosen to remove singularities at sharp corners in the real domain, for example the trailing-edge tip in the flat plate case.

Vortex motion in explicit geometries. Vortex trajectories, with no flow, are shown for three reasonably simple cases in figure 3. The flat plate is generated from the map $\zeta=\mathrm{i} \sqrt{z}$, as discussed above. For a cylinder (of radius $a$ ) at the end of a plate, we have transform

$$
\zeta=\mathrm{i} \sqrt{z}\left(1-\frac{a}{z}\right)
$$

Small modifications allow use of a similar map for an ellipse at the end of a plate, for example. Finally, for a thick aerofoil (in this case a rectangular one) it is typically more convenient to use the inverse map $z(\zeta)$ which, for a rectangular aerofoil of thickness $2 h$, is given by

$$
z=-\zeta \sqrt{\zeta-a} \sqrt{\zeta+a}+a^{2} \cosh ^{-1}\left(\frac{\zeta}{a}\right)-\frac{a^{2} \mathrm{i} \pi}{2}
$$

with $a^{2}=2 h / \pi$.

\section{B. Vortex sound}

\section{Acoustic radiation from a point vortex}

Trailing-edge noise is the result of vortical, hydrodynamic, boundary-layer turbulence interacting with the sharp trailing-edge, the resulting waves radiating at acoustic frequencies, audible as noise in the far-field. This is most noticeable when one considers the total enthalpy $B$, defined as

$$
B=\int \frac{\mathrm{d} p}{\rho}+\frac{|\mathbf{u}|^{2}}{2} .
$$

This is an apt choice of acoustic variable for two reasons [22, 23]. First, far-field pressure is simply $p=\rho_{0} B$ if the flow is at rest in the far field. Second, $B$ satisfies a forced wave equation

$$
\left[\frac{D}{D t}\left(\frac{1}{c^{2}} \frac{D}{D t}\right)-\frac{1}{\rho} \nabla \cdot(\rho \nabla)\right] B=\frac{1}{\rho} \nabla \cdot(\rho \boldsymbol{\omega} \times \mathbf{u}) .
$$




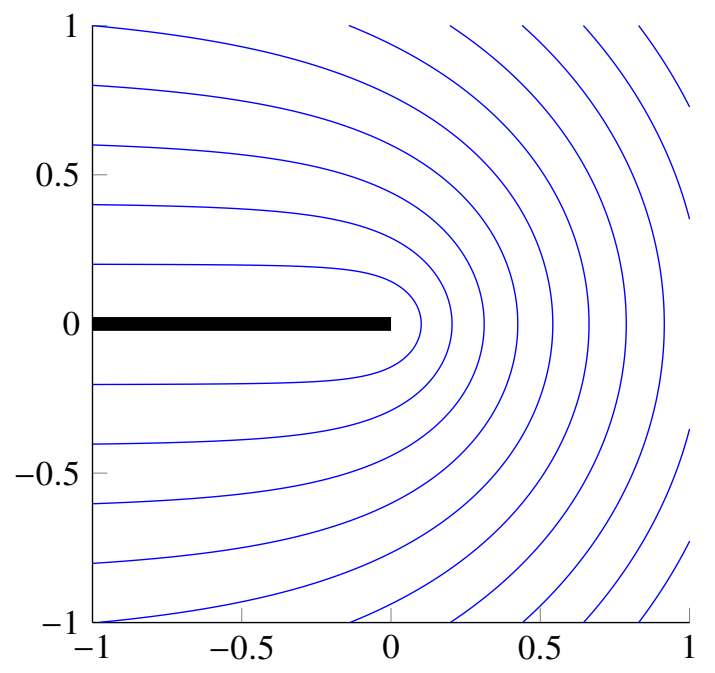

(a) Flat plate

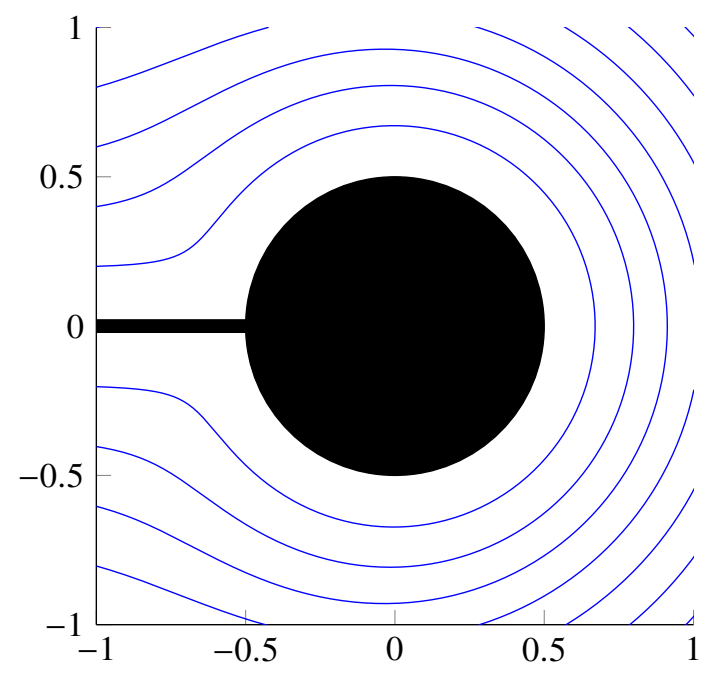

(b) Cylinder at end of plate



(c) A rectangular thick edge

Fig. 3 The path of a line vortex past the trailing-edge of an object, with no background flow. All plots have vortex strength $\Gamma=1$ with trajectories beginning at the top-left corner of the image. 
In regions of irrotational flow, the right-hand side vanishes. In particular, if we consider the earlier setup of a line vortex, the forcing is confined to a point. Constructing a Green's function for the convected wave equation, and evaluating it in the far-field, then suffices to determine far-field enthalpy (and thus pressure) from a point vortex. If we consider low Mach number flow, we need only consider the Green's function

$$
\left(\frac{1}{c_{0}^{2}} \frac{\partial^{2}}{\partial t^{2}}-\nabla^{2}\right) G=\delta\left(x_{1}-y_{1}\right) \delta\left(x_{2}-y_{2}\right) \delta(t-\tau) .
$$

There are two cases that simplify things. First, if the object itself, for example an aerofoil, is acoustically compact, in that all length scales associated with the object is much smaller than the acoustic wavelength of interest, the Green's function, in the far-field, essentially considers this object a point source. Secondly, if the object is thin yet long with respect to the acoustic wavelength of interest, for example the trailing-edge problems of interest, the Green's function can be computed as a modification to the exact Green's function for the half-plane problem, with an infinitely thin sheet. It is the latter that shall be considered explicitly. We consider the harmonic form of [17), with acoustic wavenumber $k_{0}=\omega / c_{0}$ for some well defined frequency. If we suppose the object has a streamwise lengthscale (for example the chord length) $L$, and a vertical lengthscale $\delta$ (for example aerofoil thickness) we can explicilty assume the pair of limits $k_{0} \delta \ll 1$ and $k_{0} L \gg 1$ : on the scale of acoustic waves (with length $k_{0}^{-1}$ ) the aerofoil appears long and thin. The far-field Green's function (for observer location $|\mathbf{x}| \gg$ source location $|\mathbf{y}|$ ) can be obtained by expansion in $k_{0}|\mathbf{y}|$, assumed small (so that the source is near the plate). In this far-field,

$$
G\left(\mathbf{x}, \mathbf{y} ; k_{0}\right) \sim-\frac{\phi^{*}(\mathbf{x}) \Phi^{*}(\mathbf{y})}{\pi|\mathbf{x}|} e^{i k_{0}|\mathbf{x}|} .
$$

Here, $\Phi^{*}$ and $\phi^{*}$ are potentials; in cylindrical coordinates, $\phi^{*}=\sqrt{r} \sin (\theta / 2)$ and $\Phi^{*}$ is the solution to Laplace's equation, with no-penetration on the (thick) aerofoil surface, such that $\Phi^{*} \rightarrow \phi^{*}$ as $r \rightarrow \infty$ (so that $\Phi^{*}=\phi^{*}$ in the thin-plate limit). Again, equipped with a conformal map $\zeta(z)$, we can write $\Phi^{*}=\operatorname{Re}(-\zeta(z))$, where $z=y_{1}+i y_{2}$ in this case. This can then be integrated against the right-hand side of [17], which gives

$$
\begin{aligned}
p^{\prime}(\mathbf{x}) & \sim \rho_{0} \int_{V} G(\mathbf{x}, \mathbf{y}) \nabla_{y} \cdot(\omega \times \mathbf{u})(\mathbf{y}) \mathrm{d} \mathbf{y} \\
& =-\frac{\rho_{0} \phi^{*}(\mathbf{x})}{\pi|\mathbf{x}|} e^{i k_{0}|\mathbf{x}|} \int_{V} \frac{\partial \Phi^{*}(\mathbf{y})}{\partial \mathbf{y}}(\omega \times \mathbf{u}) \mathrm{d} \mathbf{y}
\end{aligned}
$$

with $V$ the region outside the thick trailing-edge. With a point source forcing at $z_{0}(t)$, this gives

$$
p^{\prime}(\mathbf{x}, \mathbf{t})=-\frac{\rho_{0} \sin (\theta / 2)}{\pi \sqrt{r}}\left[\Gamma \operatorname{Im}\left(\left.\frac{\partial \zeta}{\partial z}\right|_{z_{0}(t)} \frac{\mathrm{d} z_{0}}{\mathrm{~d} t}\right)\right]_{t-r / c_{0}},
$$

where we have reinserted time-dependence by inverting the harmonic Fourier transform, which simply gives of the vortex noise at the retarded time $t-r / c_{0}$. The quantity in square brackets, which we identify as a sound signature of the moving vortex, is shown in figure 4 for two of the configurations being explicitly considered, both without and with background flow.

It is worth noting that this far-field acoustic pressure signature can be defined as

$$
P\left(z_{0}\right)=\Gamma \operatorname{Im}\left(\left.\frac{\partial \zeta}{\partial z}\right|_{z_{0}(t)} \frac{\mathrm{d} z_{0}}{\mathrm{~d} t}\right)
$$

with $\mathrm{d} z_{0} / \mathrm{d} t$ a function of $z_{0}$ alone through the vortex motion equation (4). That is, the far-field pressure fluctuations observed by a moving vortex can be written down purely as a function of the vortex location $z_{0}\left(=y_{1}+\mathrm{i} y_{2}\right)$, in turn purely as a function of conformal map $\zeta(z)$ (or its inverse). The three geometries for which trajectories were considered in figure 3 are revisited in figure 5 , with the noise signature emitted by a vortex at location $z_{0}$, in the absence of background flow, displayed. The primary source of noise is sharp corners, with vortices passing close to sharp corners dominating the acoustic signature (with $P$ itself being singular at these corners, the strength related to the angle of the corner). 




(a) $U=0$

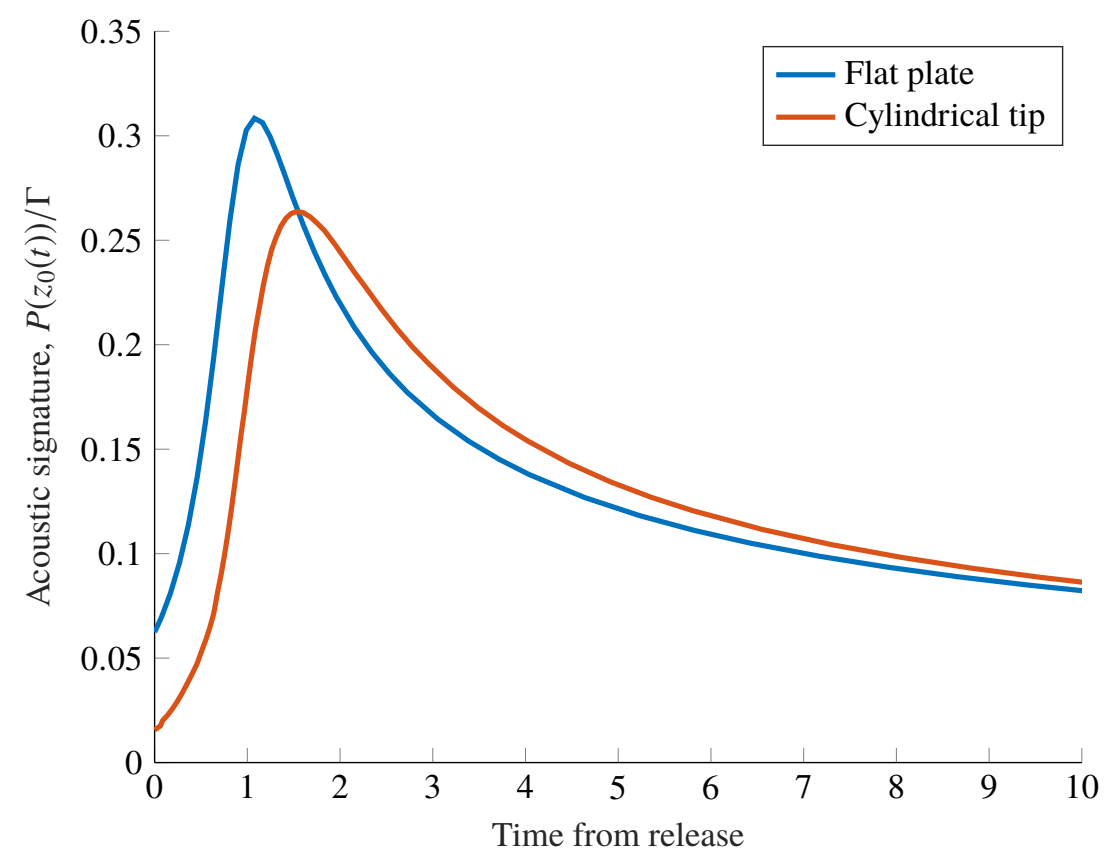

(b) $U=1$

Fig. 4 The pressure signature, $\operatorname{Im}\left(\mathrm{d} \zeta / \mathrm{d} z \times \mathrm{d} z_{0} / \mathrm{d} t\right)$, for vortices passing a flat or cylindrically tipped plate. Figure 4 a vortices following the path in figure 3, with no background flow, whereas figure $4 \mathrm{~b}$ has unit background flow (and so the vortices detach and move downstream). 


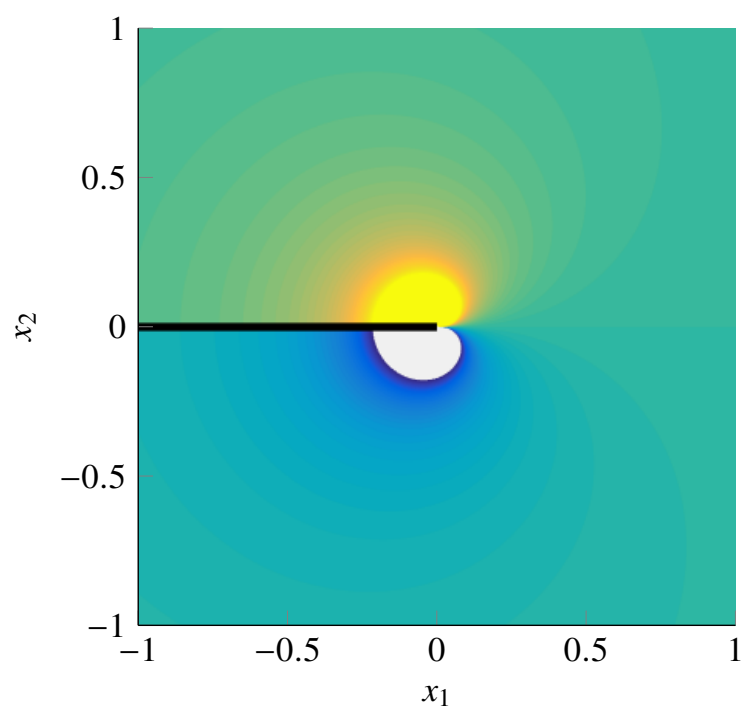

(a) Flat plate

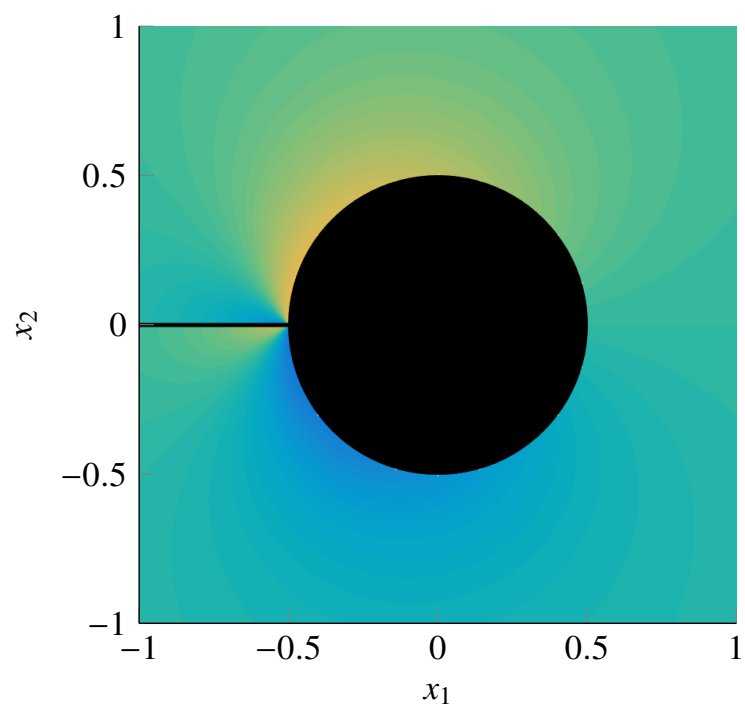

(b) Cylindrically tipped flat plate

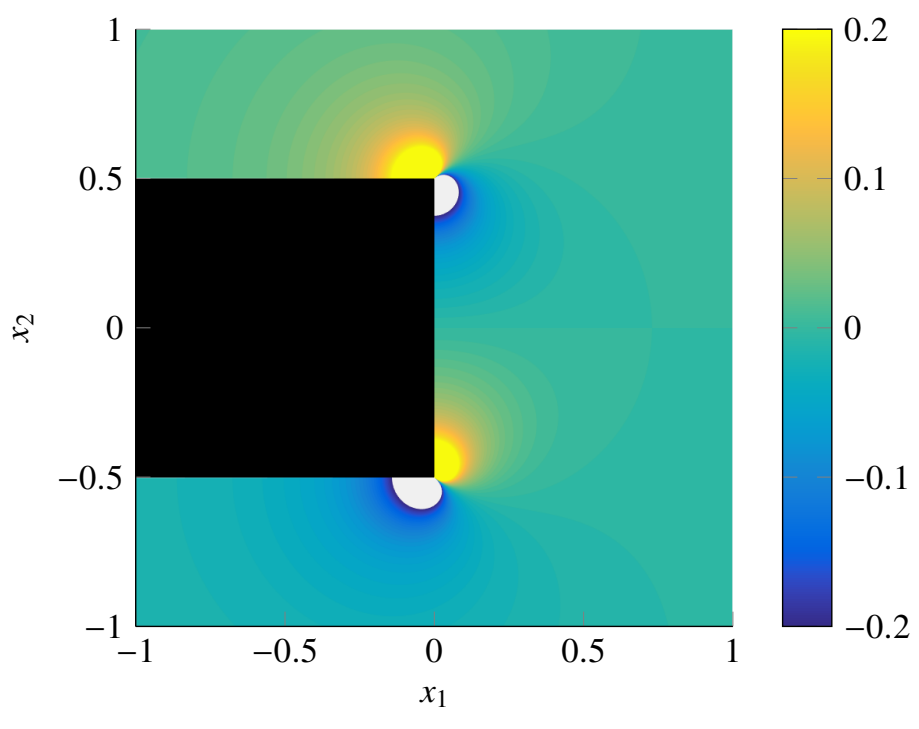

(c) Thick rectangular plate

Fig. 5 The far-field acoustic signature, $P\left(z_{0}\right) / \Gamma$ as a function of vortex location for various geometries, in the absence of background flow. In all cases the colour scale is the same, with brighter colours indicating stronger sources of sound. The acoustic signature for a vortex at a sharp corner is infinite, and for that the colour scale of the plot is truncated in the cases of the sharp trailing-edge and rectangular corners, allowing comparison between these plots and the comparatively weaker signature in the cylindrical case. 


\section{Unsteady loading on the plate}

It is possible to write (20) in terms of the unsteady loading on the plate. As per Howe [21], in terms of the enthalpy gradient on the plate, the far-field sound is given by

$$
p^{\prime}(\mathbf{x}, t) \approx-\frac{\rho_{0} \phi(\mathbf{x})}{\pi|\mathbf{x}|} \oint \Phi^{*}(\mathbf{y}) \frac{\partial B_{I}}{\partial n}(\mathbf{y}, t) \mathrm{d} S
$$

integrating around the aerofoil itself, and the enthalpy $B_{I}$ is the solution if the aerofoil did not exist. The integrand can thus be related to the unsteady loading on the plate, due to some vorticity. For a point vortex, $B_{I}=-\partial \phi_{I} / \partial t$, where the free velocity potential for a vortex $\phi_{I}$ is

$$
\phi_{I}(\mathbf{x}, t)=\operatorname{Re}\left(\frac{\Gamma}{2 \pi i} \log \left(z-z_{0}\right)\right) .
$$

Then we have, with $\Phi^{*}=-\operatorname{Re}(\zeta)$,

$$
p^{\prime}(\mathbf{x}, t) \approx-\frac{\Gamma}{2 \pi i} \frac{\rho_{0} \phi(\mathbf{x})}{\pi|\mathbf{x}|} \operatorname{Re}\left[\frac{\mathrm{d} z_{0}}{\mathrm{~d} t} \oint_{S} \frac{\zeta \mathrm{d} z}{\left(z-z_{0}\right)^{2}}\right]
$$

with integration in the anticlockwise direction in the $z$-plane about the rigid surface of the aerofoil. Whilst this does not change our expression for far-field sound (as can trivially be seen by deforming the contour to infinity, leading to only a pole contribution from $z_{0}$ ), it allows determination of where the sound emanates from, by subdividing the integration contour $S$.

\section{The Kutta condition}

The above models for vortex motion do not take into account the steady Kutta condition, although the unsteady Kutta condition [27] (regulating the acoustic singularity at the trailing-edge tip) is taken into account through the choice of Green's function (for small Mach numbers). The Kutta condition for steady flow requires the removal of velocity singularities at corners, essentially incorporating the effects of viscosity at localised points in the flow.

The flows we have derived are regular in the $\zeta$ plane, that is the upper-half plane under transformation. However, the flow field in the $z$-plane (given by the real and imaginary parts of the conjugate of $\mathrm{d} W / \mathrm{d} z=\mathrm{d} W / \mathrm{d} \zeta \times \mathrm{d} \zeta / \mathrm{d} z$ ) is singular at points where $\zeta^{\prime}(z)$ is singular, namely corners in the $z$-plane. A common method for dealing with this problem is by allowing point vortices to be released from corners. By modelling a vortex sheet being release from a corner as a concentrated core, at location $z_{j}$, with time-dependent strength $\Gamma_{j}(t)$, connected via a branch cut (a vortex sheet) to the trailing-edge, we have a simple model of vortex shedding. The vortex detaches from the trailing-edge when $\dot{\Gamma}$ becomes negative, for physical reasons. After this, it moves in the flow as a normal point vortex, with constant strength, with another vortex created at the corner, to again account for the singularity. The Brown-Michael equation [28--30] (with enhancements and acoustic applications discussed by Howe [31]) for the motion of this vortex sheet, released from corner $z_{j 0}$ with core at $z_{j}$ is

$$
\frac{\mathrm{d} z_{j}}{\mathrm{~d} t}+\left(z_{j}-z_{j 0}\right) \frac{1}{\Gamma_{j}} \frac{\mathrm{d} \Gamma_{j}}{\mathrm{~d} t}=\frac{\mathrm{d} W_{j}^{*}}{\mathrm{~d} z}
$$

with $W_{j}$ the complex velocity potential with the singularity at the vortex core removed. Inclusion of this term has a large effect on the far-field pressure signature (which must take into account the contribution from multiple vortices), with the half-plane case having been considered previously [21, 32], both qualitatively and quantitatively. Qualitatively, additional vorticity being shed from the trailing-edge is going to reduce far-field sound, as it will result in a second vortex, of opposite sign, being released. Thus vortex shedding should ideally be taken into account in determining far-field noise.

\section{Inclusion of passive flow control}

Here we consider a streamwise-orientated passive flow control device, for example the finlets investigated by Clark et al. [33]. Taking a spanwise average of the flow across these finlets allows construction of a quasi-two-dimensional model, onto which we can apply the conformal mapping techniques discussed above. We can then focus on treating the device as a porous object, subject to conditions dealt with below, as per Clark et al. [5]. The reduction to two dimensions is non-trivial, particularly in this porous region, as we have hitherto been considering a line vortex of infinite span. This 
condition can be relaxed using the construction of a vortex streak [26], which essentially allows consideration of a point vortex.

\section{A. Fences and finlets}

Finlets were introduced by Clark et al. [34], as a development of previous noise-control devices. The upper coating of an owl wing is covered in soft, downy hairs [35] which have a non-negligible contribution to their near silent flight [36]. Clark et al. [33] attempted to mimic the canopy-like effect of these hairs with direct canopy like structures and with flow-aligned parallel fibres, which were shown to vastly reduce surface pressure fluctuations underneath them. This reduction in surface pressure suggested an application as a passive noise control device, which lead to the development of finlets: long, thin flow-orientated fence-like structures, chosen to approximate the fibres.

The devices considered in [34] typically have a large aspect ratio, with a length of the order of $100 \mathrm{~mm}$ (around $10 \%$ of the experimental chord-length). By contrast, they have a small height of between 2 and $16 \mathrm{~mm}$, representing between $10 \%$ to $100 \%$ of the boundary-layer thickness. The fences typically extend some distance beyond the trailing-edge, with $10 \mathrm{~mm}$ primarily considered experimentally, though the no-extension case was also considered. The structures were repeated spanwise, with typical spanwise thickness $0.5 \mathrm{~mm}$, though thicker devices were considered. Importantly, the spacing between the devices was varied, between 1 and $10 \mathrm{~mm}$. This, coupled with the finlet thickness, gives a fractional open area, discussed below, of $0.5<\beta<1$. This device is placed at the end of the DU96-W180 section (a standard, reasonably thin, wind-turbine blade profile) in a flow with freestream velocity ranging from $12 \mathrm{~m} \mathrm{~s}^{-1}$ to $57 \mathrm{~m} \mathrm{~s}^{-1}$

This section tries to determine whether this spacing can be optimised using simple mathematical models. Primarily focusing on the somewhat abstract case of a circular device, with radius $a$ corresponding to finlet height and free-stream velocity $U$ chosen as above. All length and time scales are scaled with respect to these quantities. More realistic geometries will be discussed (for example an elongated ellipse immitating the aspect ratio of the finlets). Further, some discussion will be given of moving the device upstream, as has been investigated experimentally [8]. Whilst this upstream configuration in practice results in sound reduction, we shall see that the argument in this section cannot easily predict it. There is, however, a good agreement between the model and experiment when the device is at the trailing-edge, with the model qualitatively predicting noise reduction in all cases it is observed experimentally.

\section{B. A point vortex, or vortex streak}

Difficulties arise when a third dimension is included, in that the idealised model of a line vortex (infinite in the direction of the coordinate vector $\mathbf{e}_{3}$ ) cannot be directly generalised when the domain is spanwise inhomogeneous (for example, a flow passing over finlets or other spanwise-varying noise control mechanisms). Previously, Howe [22] dealt with this problem by considering the Rapid Distortion Theory limit, in which the flow speed $U$ is much greater than vortex strength $\Gamma$ and so the nonlinear vortex self-interaction can be neglected. An infinite line vortex upstream then propagates the the (fully three-dimensional) background flow, which distorts the line vortex. Whilst this is an adequate generalisation of the problem, it moves discussion rapidly from analytical considerations to purely computational ones, and it is difficult to apply a Kutta condition to the resulting three-dimensional problem.

An alternative method, as suggested by Glegg and Devenport [26], is to consider a vortex streak. Suppose we have a closed vortex loop, with two legs a distance $\epsilon$ apart, as indicated in Figure 6 Since this is a closed loop, the strength of the filament along the loop is constant, $\Gamma$ [37]. For $\epsilon$ small, the acoustic contribution from each leg of the loop, contained within the Lamb vector $\omega \times \mathbf{u}$, cancels out, leaving contributions only from the leading and trailing edges of the loop (which need not cancel). Either the loop extends to infinity upstream or one considers the ends far enough apart such that they are statistically independent in turbulent flow, but either way this allows consideration of the single vortical quantity

$$
\omega=\epsilon \Gamma \hat{\mathbf{s}} \delta\left(\mathbf{x}-\mathbf{x}_{0}\right) .
$$

This describes a point vortex orientated in the $\hat{\mathbf{s}}$ direction. In general, we consider $\hat{\mathbf{s}}=\mathbf{e}_{3}$, the spanwise unit vector. This infinitesimal construction allows relaxation of the assumption of spanwise homogeneity, as we can construct any vortex line (which need not be straight) from integration of such elements.

\section{Channelling flow}

We make the assumption that the blocking object does not distort the background flow greatly, instead simply channelling it (and thus accelerating it), which has some justification from numerical experiments [9]. Suppose (as in figure 7) the finlets repeat on lengthscale $d$, and the gap between two finlets is $s$ (so that the width of each finlet is $d-s$, 


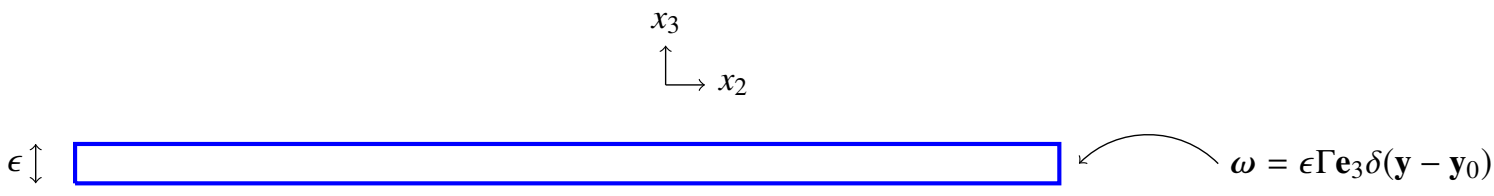

Fig. 6 A vortex streak

and is small compared with $d$ ). Defining the open area $\beta=s / d \in[0,1]$, we posit the following boundary conditions at the interface between porous (superscript $(i)$ ) and open (superscript $(o)$ ) media

$$
\begin{aligned}
\mathbf{n} \cdot \mathbf{u}^{(o)} & =\beta \mathbf{n} \cdot \mathbf{u}^{(i)}, \\
\mathbf{n} \times \mathbf{u}^{(o)} & =\mathbf{n} \times \mathbf{u}^{(i)},
\end{aligned}
$$

respectively stating that the flow into the porous object is accelerated, whereas the flow tangential to the object is unchanged. The normal is defined as pointing out of the porous object (from $V^{(i)}$ to $V^{(o)}$, respectively the partially-open domain and the fully open domain). At rigid surfaces, $\mathbf{n} \cdot \mathbf{u}=0$.

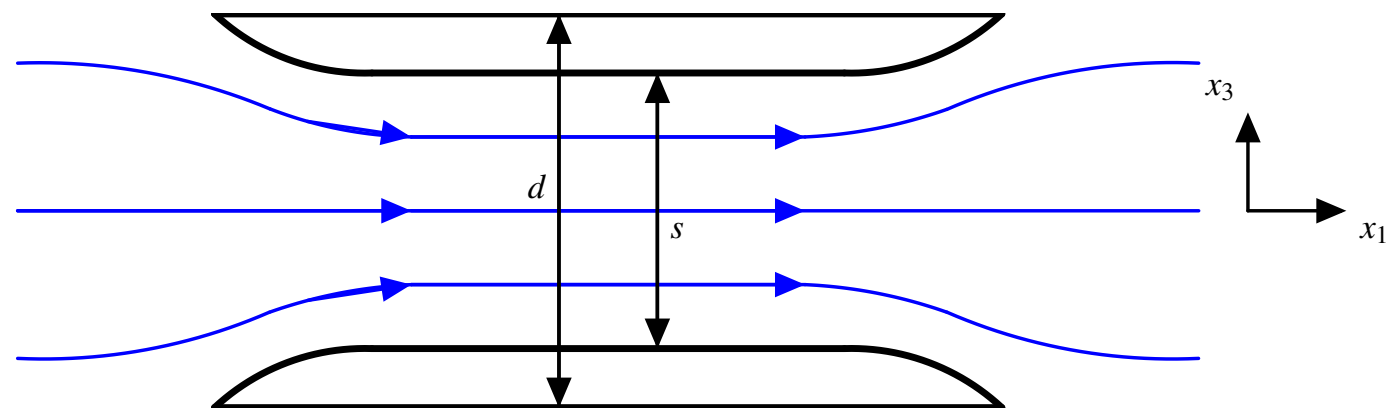

Fig. 7 The assumption of channelling flow - other than a spanwise compression of flow, there is otherwise no effect on the flow through the passive devices. The two dimensions of interest are shown: the gap between the devices $d$ and the spacing within the devices, $s$.

This quasi-two-dimensional problem can be solved through the use of a complex potential, $W=\phi+\mathrm{i} \psi$, for which the boundary conditions can be recast. Defining the outer potential $W^{(o)}$ and the inner $W^{(i)}$, the boundary conditions on the porous boundary are

$$
\begin{aligned}
\operatorname{Re}\left(W^{(o)}-W^{(i)}\right) & =0 \\
\operatorname{Im}\left(W^{(o)}-\beta W^{(i)}\right) & =0
\end{aligned}
$$

Certain domains lends themselves neatly to the solution of this problem in the steady flow case, in particular the flow through a porous cylinder (which may or may not be at the end of a flat plate, since the flow is presumed aligned with the plate). In this case, we have complex velocity potentials

$$
\begin{aligned}
& W^{(o)}(z)=U\left[z+\frac{1-\beta}{1+\beta} \frac{a^{2}}{z}\right] \\
& W^{(i)}(z)=U z \cdot \frac{2}{1+\beta}
\end{aligned}
$$

where $a$ is the radius of the cylinder in question, and $z=x_{1}+\mathrm{i} x_{2}$. The velocity potential and streamfunction of this (irrotational) background flow are the real and imaginary parts of $W$ respectively. It is relatively straightforward to check that the continuity conditions are satisfied at $r=a$, and the resulting lines of constant $\phi$ and $\psi$ are displayed in figure 8 .

This can be generalised to somewhat more complicated domains, via suitable conformal maps. If $z\left(z_{2}\right)$ is a complex function mapping a complicated boundary in the $z_{2}$ plane to the exterior of the unit circle, then the expression for 




(a) Velocity potential

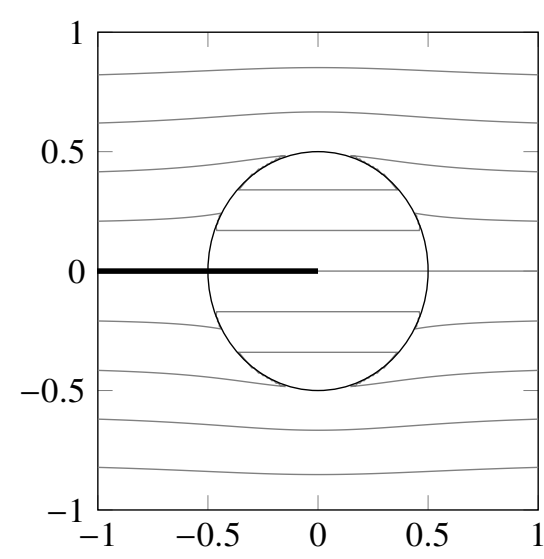

(b) Streamfunction

Fig. 8 Velocity potential and streamfunction for a flow through a mostly-open cylinder, at the end of a flat plate. Here, $\beta=0.7$. Streamlines are moved away from the trailing-edge of the plate and the flow is accelerated through the channel.

uniform flow through an partially porous object with the aforementioned complicated boundary is given by (29) with $z=z\left(z_{2}\right)$. This clearly satisfies the boundary conditions in complex form, and that the map is conformal ensures Laplace's equation is still valid.

Glegg and Devenport [26] generalised this to two concentric circles with a rigid inner circle, thereby correcting a mistake by this author [5] which claims the earlier result is more generic. However, provided the map $z\left(z_{2}\right)$ is real on the real axis, and thus preserves it under the transformation, it is possible to consider reasonably complicated porous objects at the end of a flat plate. This restriction mostly limits to symmetric objects (across $x_{2} \gtrless 0$ ), which is expected as asymmetry might drive a vertical flow through the flat plate, which must then be corrected in turn. By way of simple example, one can consider mapping an ellipse to the unit circle, with the inverse map given by

$$
z_{2}(z)=\frac{1}{2}\left(R z+\frac{1}{R z}\right)
$$

which maps the unit circle to an ellipse with semiaxes $\left|R+R^{-1}\right|$ and $\left|R-R^{-1}\right|$, and is discussed later. It is also possible to simply move an object up and down the semi-infinite plate under $z \mapsto z-b$. This allows investigation of the realistic case of an upstream passive flow device.

Whilst, in suitable geometries, it is straightforward to calculate the behaviour of uniform upstream flow through the object, computation of the flow field due to a point vortex near the object is not trivial. This makes the inclusion of vortex self-motion within the model difficult. Further, the inherently three-dimensional nature of a vortex streak further reduces practicality of self-motion within the quasi two-dimensional framework. However, in realistic flows we expect background flows to be much larger than the vortex strength, except perhaps near stagnation points of the background flow. Hence, under this assumption that $U \gg \Gamma$, the basic assumption of Rapid Distortion Theory, we can neglect the vortex self motion term. Rapid Distortion Theory (RDT) assumes that the flow develops on a timescale that is much slower than the convective timescale: the vortex is convected with the mean flow and nonlinear effects are ignored.

\section{Sound reduction: A centreline vortex through a partially-open cylinder}

Consider the simplest case of a point vortex (derived from a vortex streak) moving along the spanwise centreline of a fence-like noise control device (that is, equally spaced between fences), moving with the background flow. The centreline approximation allows the reasonably aggressive acoustic assumption that it is the rigid flat plate, and not the noise-control devices themselves, that contribute to the far-field noise (with fence contributions essentially cancelling). 


\section{Vortex motion}

In the RDT limit, the vortex moves along streamlines of the background flow. For uniform flow through a cylinder with open area $\beta$, we have

$$
\psi= \begin{cases}U\left(x_{2}-\left(\frac{1-\beta}{1+\beta}\right) \frac{a^{2} x_{2}}{x_{1}^{2}+x_{2}^{2}}\right) & \left(x_{1}^{2}+x_{2}^{2}\right)>a^{2} \\ \frac{2}{1+\beta} \cdot U x_{2} & \left(x_{1}^{2}+x_{2}^{2}\right)<a^{2}\end{cases}
$$

As we shall see, the noise signature is dominated by the tip $x_{1}=0$. A vortex released at upstream vertical position $y_{\infty}$ passes $x_{1}=0$ at

$$
y_{2}=\frac{y_{\infty}}{2}+\sqrt{\left(\frac{y_{\infty}}{2}\right)^{2}+a^{2} \frac{1-\beta}{1+\beta}}
$$

when the release position $y_{\infty}>2 \beta a /(1+\beta)$. Conversely, if $y_{\infty}<2 \beta a /(1+\beta)$, the vortex moves into the porous object and passes the trailing-edge at

$$
y_{2}=\frac{\beta+1}{2 \beta} y_{\infty} .
$$

These combine to give a continuous function of release location $y_{\infty}$.

\section{Vortex sound}

Upon the exclusion of self-motion, the vortex sound of a vortex at $z_{0}=y_{1}+\mathrm{i} y_{2}$ is quantified by acoustic signature

$$
P\left(z_{0}\right)=\Gamma \operatorname{Im}\left(\left.\frac{\partial \zeta}{\partial z}\right|_{z_{0}(t)} \frac{\mathrm{d} z_{0}}{\mathrm{~d} t}\right)=\Gamma \operatorname{Im}\left(\left.\left.\frac{\partial \zeta}{\partial z}\right|_{z_{0}(t)} \frac{\mathrm{d} W^{*}}{\mathrm{~d} z}\right|_{z_{0}(t)}\right) .
$$

For a vortex at the centreline, we approximate the acoustic Green's function as seeing only the flat plate within the partially-open object, and thus take $\zeta=\mathrm{i} \sqrt{z}$. Recalling that reducing the spanwise length of the vortex element to fraction $\beta$ simply reduces the total strength by $\beta$, it is important to include an extra factor of $\beta$ when the vortex is within the partially open object, so that $\Gamma$ is replaced by $\beta \Gamma$ in the above expression.

The relative noise as $\beta$ is varied indicated in figure 9 is from a vortex released from upstream $y_{\infty}=0.1 a$ and passing through the porous object, along a streamline of the background flow. The acoustic signature is dominated by a peak near $y_{1}=0$, as suggested above. This noise is the result of the proximity of the vortex to the trailing-edge.

For a vortex at $y_{1}=0$, the acoustic signature $P$ can be analytically determined. Assuming the vortex is within the porous object, we have

$$
P=\frac{1}{2 \sqrt{2}} \frac{U \Gamma}{\sqrt{y_{2}}} \frac{2 \beta}{1+\beta}
$$

by direct evaluation of $\mathrm{d} W / \mathrm{d} z$ at $z_{0}=\mathrm{i} y_{2}$. We can relate $y_{2}$ to the upstream release point via (33), which gives, as a function of release point and open area $\beta$

$$
P\left(y_{\infty}, \beta\right)=\frac{U \Gamma}{2 \sqrt{2 y_{\infty}}}\left(\frac{2 \beta}{1+\beta}\right)^{3 / 2} .
$$

Whilst in practice the peak is not at $y_{1}=0$, but slightly beyond it, this provides a reasonable approximation of the $\beta$-dependence of $P$ throughout the porous region, as can easily be checked numerically. Thus, we end up with an amplification factor (compared to the open object, $\beta=1$ )

$$
f(\beta)=\left(\frac{2 \beta}{1+\beta}\right)^{3 / 2}
$$

The (logarithmic) behaviour of this function is plotted in figure 10 , as the top (blue) line. We see $f(1)=1$ and $f(\beta)<1$ for $\beta<1$ : there is a reduction in far-field noise. Of course, for $\beta$ too small the effects of the noise control device in creating noise itself must be considered, and the various assumptions on channeling flow and vortex behaviour are less appropriate. 


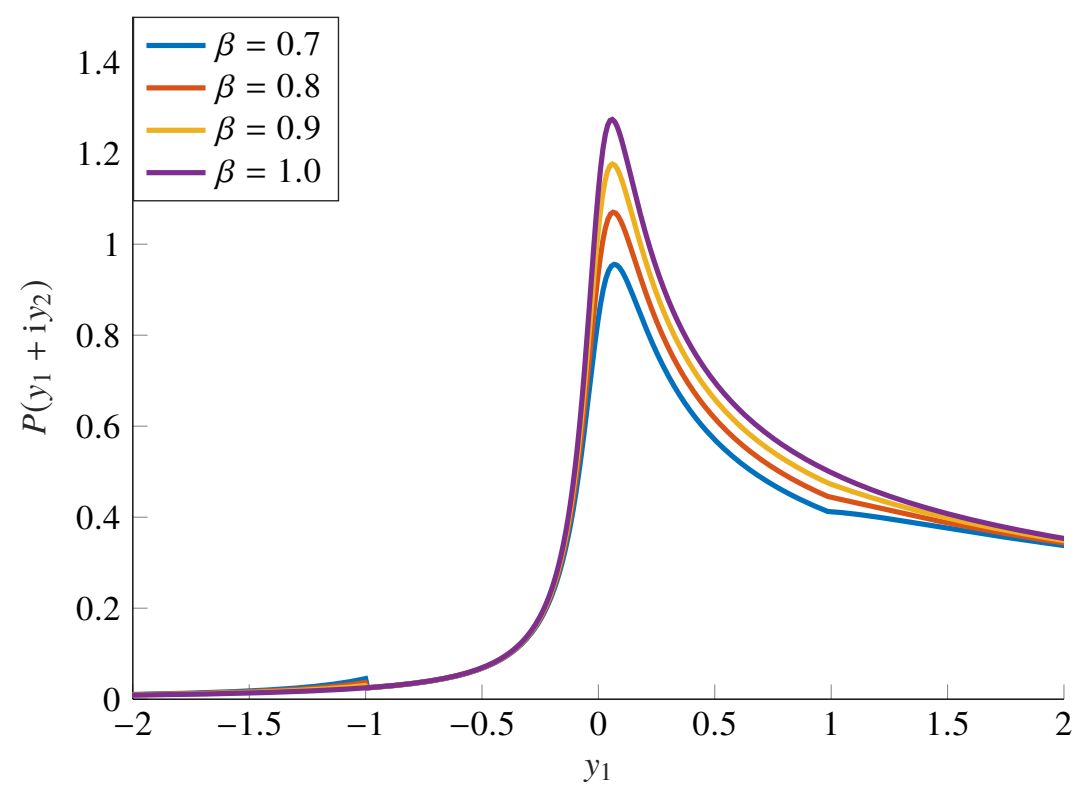

Fig. 9 A vortex released from upstream vertical position $y_{\infty}=0.1 a$ passing through a porous cylinder (with all lengths scaled by $a$, and time by $a / U$ ). The acoustic signature $P(z)$ is given, as a function of streamwise displacement $x_{1}$, for $\beta$ ranging from 0.7 to 1 (fully open). Due to displacement of the vortex from the trailingedge, noise is reduced, with the acceleration of the vortex due to the porous object canceled out by the compression of the vortex within the porous object.

This approximation quantifies the vortex noise purely by the noise peak through its passage. More complicated norms can be considered, for example (46) will consider a variety of measures of the total noise signature of a vortex as it passes the trailing-edge, including the integrated Sound Pressure Level. However, the reasons for not doing so in this case are twofold: firstly, the ratio of peak height is a good proxy for the ratio of far-field pressure magnitude throughout passage. This is readily checked: whilst $f(\beta)$ is the ratio at $y_{1}=0$, it corresponds closely to the ratio of the maxima, even if absolute values differ substantially.

Secondly, we have discounted the effect of vortex shedding. Whilst in the RDT approximation this is appropriate as far as vortex paths are concerned, the inclusion of concentrated vorticity shedding from the trailing-edge will have an effect on the generation of far-field noise, since a shed vortex will create its own noise signature. As discussed by Howe [22] this has the tendency to reduce far-field noise, as the shed vortex will have opposite sign and thus cancel out the incident vortex. This is most apparent in the tail of a plot of $P$ against vortex location or time, with the long-time noise signature vanishing, though there is comparatively little effect on the noise peak itself. Whilst this ensures the conclusions of sound reduction, and the scaling factor $f(\beta)$, are appropriate, it does preclude using an integrated norm across the passage of the vortex, due to this deviation for large times from the physically appropriate result.

This result does not depend on the device size, $a$, since it cancels out of the vortex trajectory when the vortex enters the device. This implies a uniformity to the scaling factor regardless of the initial location of the vortex, provided the vortex is released upstream sufficiently close to the plate so that it enters the control device.

\section{E. More realistic geometries and alternative configurations}

There are two aspects it is possible to include without major alterations to the model. To consider a large aspect-ratio blockage, we consider the flow through a partially open elliptical cylinder. The complex potential, given by

$$
W= \begin{cases}U\left[A(R, \beta)\left(z_{2}(z, R)+\frac{a^{2}}{z_{2}(z, R)}\right)+B(R, \beta) z_{2}(z, R)\right] & \left|z_{2}(z)\right|>a \\ U C(R, \beta) z & \left|z_{2}(z)\right|<a\end{cases}
$$


where $z_{2}=\frac{z}{2 R}+\frac{1}{2 R} \sqrt{z-2 a} \sqrt{z+2 a}$ maps an ellipse with semiaxes $\left|R+a^{2} / R\right|$ and $\left|R-a^{2} / R\right|$ to the circle of radius $a$, discussed previously. The leading constants $A, B$, and $C$ are somewhat more complicated, with (assuming $R>a^{2} / R$ )

$$
\begin{aligned}
& A=\frac{(1-\beta) R^{2}+(1+\beta) a^{2}}{(1+\beta) R^{2}+(1-\beta) a^{2}} \\
& B=\frac{2 \beta\left(R^{2}-a^{2}\right)}{(1+\beta) R^{2}+(1-\beta) a^{2}} \\
& C=\frac{2 R}{(1+\beta) R^{2}+(1-\beta) a^{2}}
\end{aligned}
$$

which combine to ensure the jump conditions across the ellipse, given by $\left|z_{2}(z)\right|=a$, are satisfied. The vortex trajectories, overlaid onto the noise signature (as a function of vortex location) are shown for a reasonably high aspect-ratio case in figure 11 .

If a vortex is released upstream at height $y_{\infty}$ and intersects the obstacle, it passes through the obstacle at constant height $y_{2}$, with the displacement ratio given by

$$
\frac{y_{2}}{y_{\infty}}=\frac{(1+\beta) R^{2}+(1-\beta) a^{2}}{2 R^{2} \beta}
$$

which regains the result in the circular case in the limit $R \rightarrow \infty$ and tends to unity in the limit $R \rightarrow a$ (a flat plate), both as expected. Approximating, as in 36 , the noise signature $P$ by the signature at $y_{1}=0$, the modified noise reduction factor $f(\beta ; R)$ can be determined as

$$
f(\beta ; R)=\left[\frac{2 R^{2} \beta}{(1+\beta) R^{2}+(1-\beta) a^{2}}\right]^{3 / 2} .
$$

Then for $R$ large, we regain the circular domain and the earlier result. As $R \rightarrow a$ we progressively get an ellipse of larger aspect ratio. The effect of this is only ever to decrease noise (with $f$ monotonic in $R$ ), however this only applies to vortices trapped within the device (hence it breaking down for $R=a$, where a noise reduction is predicted despite the flat plate result being reobtained).

Finally, a similar analysis could be done for an upstream noise control device, via transformation $z \mapsto z-b$ for some real $b$. This mimics a variety of behaviours previously considered [7]. If, however, the device is purely upstream of the trailing-edge, then this method shows little sound reduction. This follows from the inherent symmetry of the flow field under $x_{1} \mapsto-x_{1}$, or, in the transformed case, $x_{1}-b \mapsto b-x_{1}$. If the trailing-edge is beyond the downstream limit of the device, the irrotational flow field relaxes to its uniform counterpart and there is little change in the sound signature, dominated by the vortex near the trailing-edge. This highlights the fundamental physics missing from the model. By modelling the noise control devices purely as perturbing an irrotational flow, one vastly overestimates the scale of the object (which, in practice, would be within a rotational boundary-layer). Whilst the deflection of turbulence (and thus vortical motion) from the trailing-edge may well account for the noise reduction mechanism, a full understanding requires inclusion of sheared background flow, and the effects of separation and relaminarisation in the wake of the noise control device [8, 13]. This has the same fundamental effect: upstream turbulence is moved further from the trailing-edge, and thus weakens the coupling between hydrodynamic vorticity and far-field acoustic noise, but this effect cannot be replicated in this simple model.

\section{Inclusion of active flow control}

We now instead consider the inclusion of active flow control to Howe's trailing edge model [21], mimicking the experimental set up of [13]. We consider a point vortex moving in background velocity field $(u, v)$ due to a mean flow and the flow induced by an active section upstream of the trailing edge.

\section{A. Active Flow Model}

Here we introduce the active flow model as illustrated in figure 12 


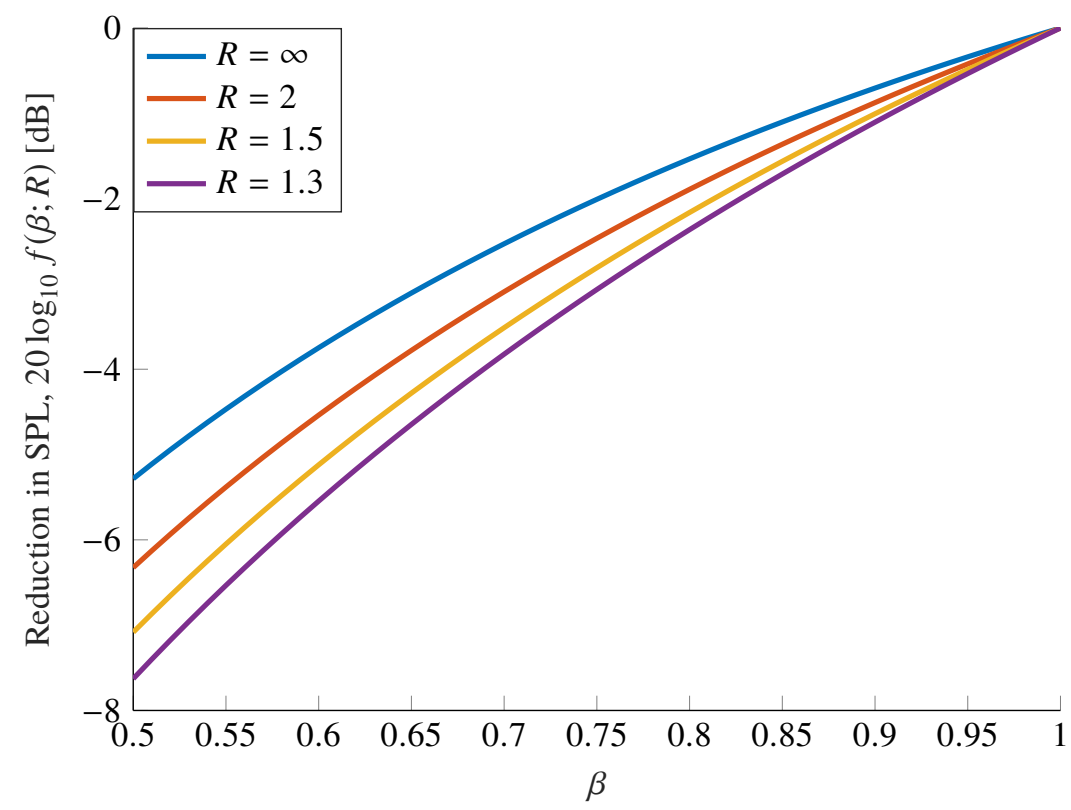

Fig. 10 The behaviour of the amplification factor $f(\beta ; R)$ as $\beta$ is varied, with $20 \log _{10} f(\beta ; R)$ plotted to show noise reduction. In the limit $R \rightarrow \infty$, we obtain the circular result, which shows for reasonably small blockages, for example $\beta \sim 0.7$, there is a predicted 2.5 decibel noise reduction. Increasing the aspect ratio of the elliptic blockage ( $R \rightarrow 1$, with $a$ scaled to 1$)$, further reduces the noise for all $\beta$.

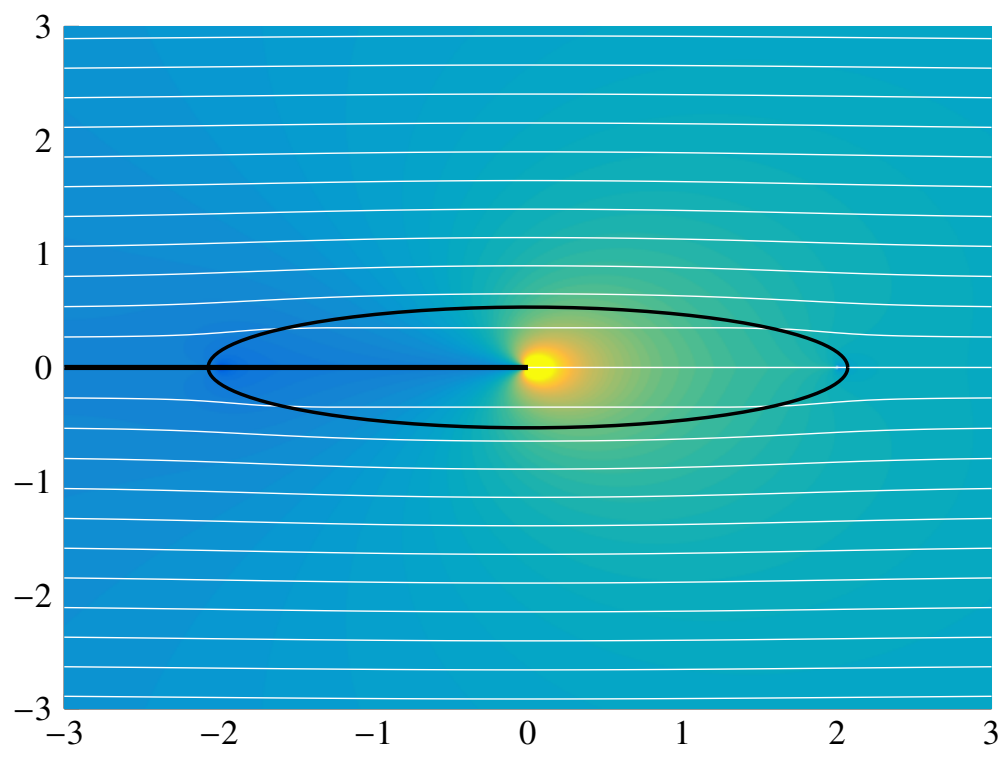

Fig. 11 The paths of vortices through a porous ellipse, following streamlines of the background flow. The acoustic signature $P$ is plotted underneath, showing a strong noise source for vorticies near the tip, reduced by the deflection of vortices away from the tip, with a secondary reduction coming from the weakening of the vortices within the object itself. For this example, $\beta=0.7$ and $R=1.3$ (corresponding to semiaxes of 2.1 (horizontal) and 0.53 (vertical), with undisturbed flow speed $U_{\infty}$ and object dimension $a$ both set to 1 . 


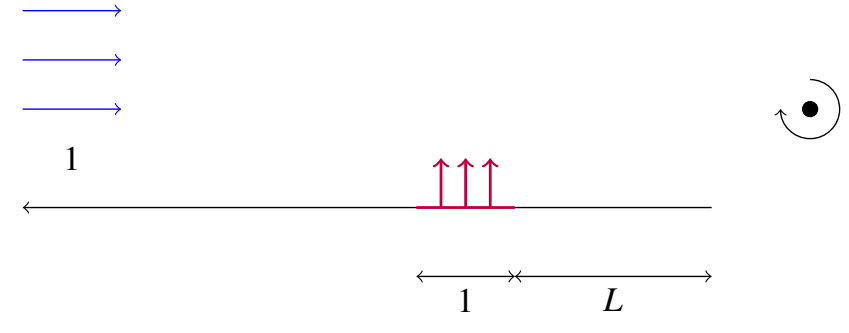

Fig. 12 Active flow modelling: point vortex interacting with a trailing edge in a background flow field

\section{Non-dimensionalisation}

We choose to non-dimensionalise velocities with the mean stream velocity and lengths with the streamwise length of the active control section, again taking the origin to be the trailing edge. This leaves variable parameters in the initial location and strength of the incident vortex, and the location $L$ and mass flux $Q$ of the active control section. For comparison with the normal blowing experimental set up of [13] the corresponding dimensionalization values are $U^{*}=15 \mathrm{~m} \mathrm{~s}^{-1}, L^{*}=0.03 \mathrm{~m}$ and $T^{*}=0.002 \mathrm{~s}$.

For comparison with the experimental results, we must choose appropriate values for the strength and location of an incident vortex. The point vortex is taken to be initially located at $z_{\infty}=-x_{\infty}+i y_{\infty}$. We first consider a vortex with normal displacement from the plate corresponding to the location of large rms velocity fluctuations in [13], and the vortex strength to be determined so that its self-velocity at this location corresponds to the peak rms velocity fluctuation. Given these assumptions we initially take non-dimensional values of normal displacement $y_{\infty}=0.5$ and vortex strength $\Gamma=0.5$. The corresponding non-dimensional values to the active flow parameters considered in [13] are active flow location $L \approx 6$ and active flow flux $Q \approx 0.1,0.3$. Unless otherwise stated, the initial streamwise displacement of the vortex is taken to be $x_{\infty}=10$.

\section{Background flow}

The vortex sound framework (as discussed in Section II) typically considers a point vortex moving in a background potential flow. An arbitrary steady background flow such as that arising from active blowing at the trailing edge may be specified only if we additionally suppose that the point vortex is weak relative to the background flow. We thus for simplicity of applying the vortex sound framework initially seek an appropriate potential flow (as opposed to an arbitrary flow) that simulates an active control device and satisfies the steady Kutta condition at the trailing edge. The effect of a section of active flow $[a, b]$ on the upper surface of an otherwise rigid plate is modelled by imposing a specified normal velocity $v^{ \pm}$on the upper/lower boundaries of the plate:

$$
v^{-}(x)=0, \quad v^{+}(x)= \begin{cases}f(x) & a<x<b \\ 0 & \text { otherwise }\end{cases}
$$

where $f(x)$ is to be determined.

\section{Arbitrary normal velocity imposed on aerofoil}

The Plemelj formulae [38] may be used to solve for such a potential flow with arbitrary profile $f$ in integral form:

$$
(u-i v)(z)=-\frac{1}{2 \pi} \int_{a}^{b} \frac{f(\tau)}{\tau-z} \mathrm{~d} \tau-\frac{\sqrt{z}}{2 \pi} \int_{a}^{b} \frac{f(\tau)}{\sqrt{\tau}} \frac{\mathrm{d} \tau}{\tau-z}
$$

The simplest normal velocity profile is uniform, corresponding to a 'tophat' or 'box' function on plate: $f(x)=1$. In this case the solution may be written explicitly:

$$
(u-i v)(z)=\frac{1}{\pi}\left[\log \left(\frac{z-a}{z-b}\right)+\log \left(\frac{1-\sqrt{z / a}}{1+\sqrt{a / z}}\right)-\log \left(\frac{1-\sqrt{z / b}}{1+\sqrt{b / z}}\right)\right]
$$

where the branch cut of $\log$ and the square roots is taken to be the interval $[a, b]$. 


\section{Injected channel}

In order to mimic a real injected flow scenario one might explicitly model an entry channel. An inclined jet may be modelled by a set of point mass sources located within such a geometry that appropriately confines the flow. The flow for a piecewise-linear geometry may be found using conformal maps and the Schwarz-Christoffel transformation, c.f. Jaworski [24]. Whilst artificial, this model captures key features amenable to analytic investigation: the influence of blowing strength, inclination and location on far-field vortex sound.

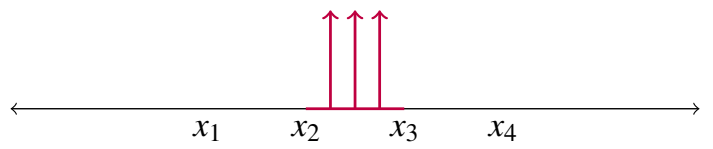

(a) $\zeta$-plane

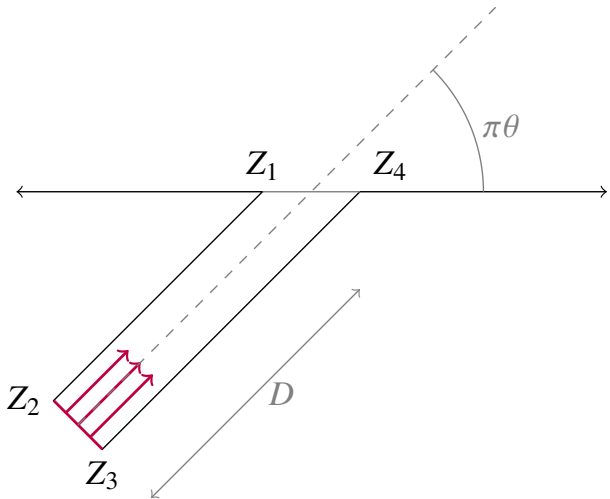

(b) Z-plane

\section{Fig. 13 Illustration of fluid injection model into the UHP from an inclined channel}

The geometry considered is visualised in figure 13 . First we obtain the flow in the UHP due to a region of point mass sources on the real axis, on which a no-penetration boundary condition is otherwise imposed (figure 13a). Next a Schwarz-Christoffel map is used to deform the real axis to imitate an inclined jet entering the UHP (figure 13b). The Schwarz-Christoffel formula [38] gives

$$
Z=K \int_{1}^{\zeta} \frac{1}{\left(\chi-x_{1}\right)^{-1+\theta}\left(\chi-x_{2}\right)^{1 / 2}\left(\chi-x_{3}\right)^{1 / 2}\left(\chi-x_{4}\right)^{-\theta}} \mathrm{d} \chi
$$

where $x_{i}$ must chosen to ensure desired relative side lengths and the constant $K$ gives an overall scaling and orientation. The appropriate map, and its inverse may be computed using the SC-toolbox package described in [39]. We note that we may choose the location of the stagnation point, which is taken to coincide with the upstream edge of the active section. A comparison of the so attained plate flow profile with more naïve profiles is presented in figure 14, along with the respective acoustic signature. We see the effect of different flow profiles is minimal on the acoustic signature.

\section{B. Active control results: vortex paths and far-field acoustic signature}

\section{Initial discussion of results}

The model provides a theoretical prediction of the displacement of an incident vortex by a given active flow flux, and the associated far-field acoustic reduction. An initial impression may be gained by considering the streamlines of the background flow with active device (figure $15 \mathrm{a}$ ) showing the displacement of a vortex in the rapid distortion limit. Additionally, the acoustic sensitivity plot (figure $15 \mathrm{~b}$ ) indicates that we can expect an additional, but weaker, acoustic contribution due to the vortex interaction with the active device.

Results for a 'box' type profile comparing different active flow rates are presented in figure 16 The vortex is clearly displaced away from the trailing edge by the flow injection, more severely for higher injection rates. We see two peaks in the acoustic signature plot (figure 16b, the first due to an interaction with the active device causes the vortex to cross the streamlines of a flow about the trailing edge, and the second due to the vortex passing the trailing edge. For parameters considered the trailing edge interaction is clearly dominant. Active flow rates of 0.1 and 0.3 lead to a reduction in peak acoustic signature of $0.73 \mathrm{~dB}$ and $1.8 \mathrm{~dB}$ respectively, and $1.7 \mathrm{~dB}$ and $4.7 \mathrm{~dB}$ in far-field power radiated. As an overall 
$f(x)=1$

$f(x)=6 x(1-x)$

channel $\theta=0.25$

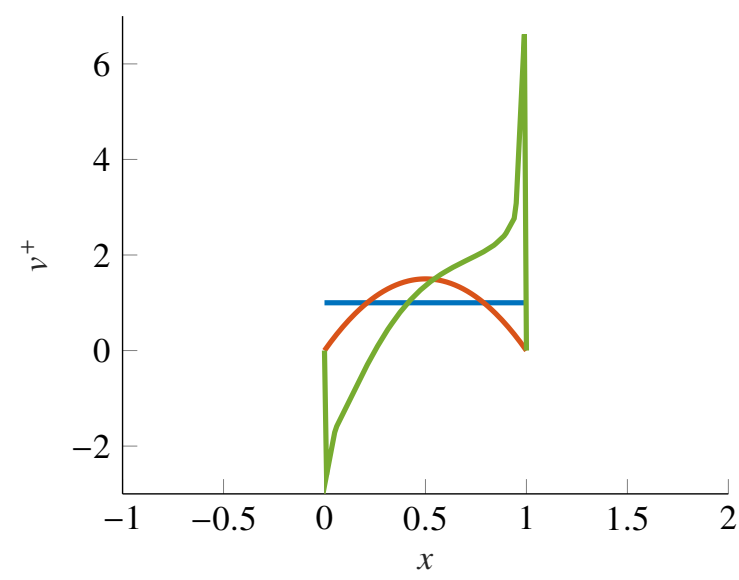

(a) Normalised active flow profile

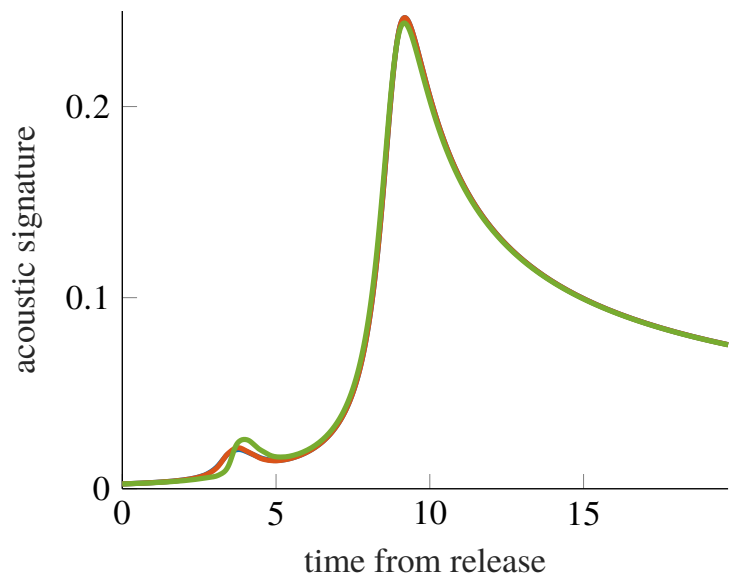

(b) Acoustic signature time history

Fig. 14 Comparison of acoustic signature $P$ varying the active flow profile on a finite section

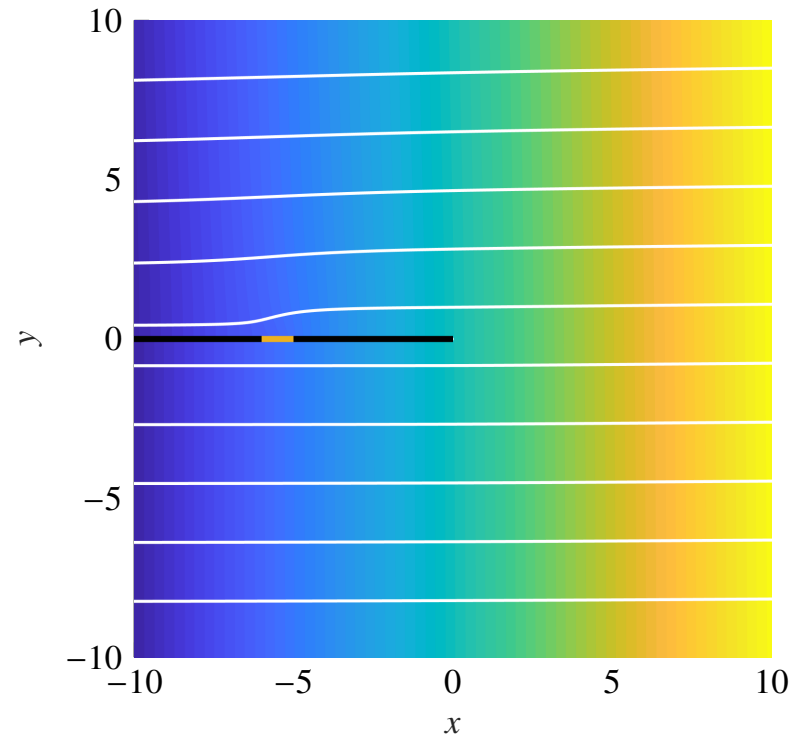

(a) Streamlines for inclusion of an active flow device.

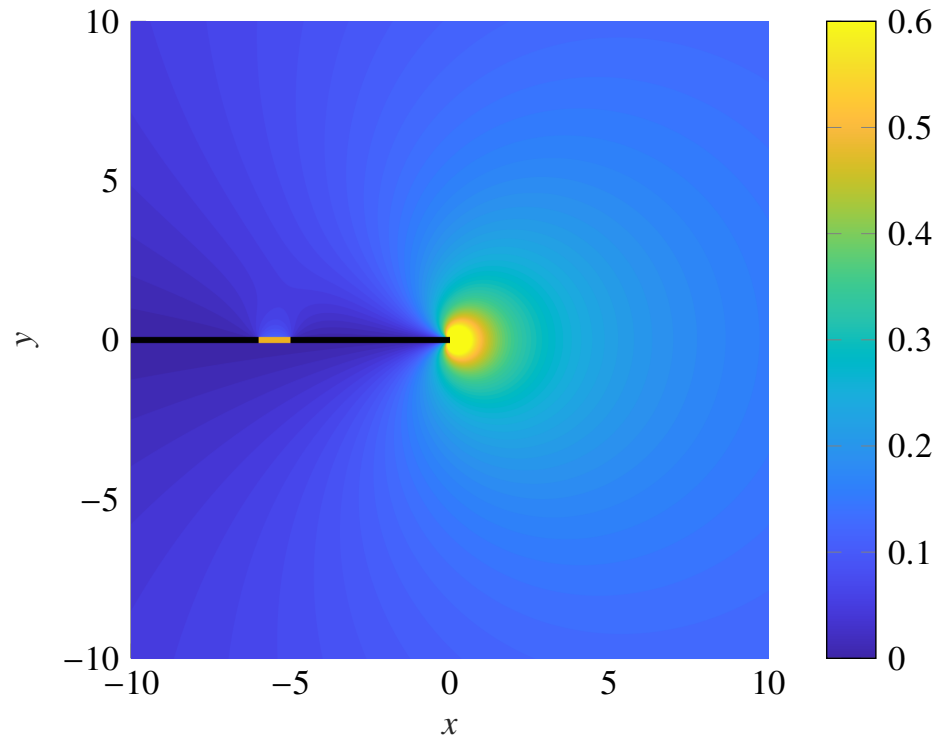

(b) Far-field acoustic sensitivity

Fig. 15 Unit mean flow and active strength 0.6 at displacement 5 from edge, in the RDT limit. Inclusion of vortex-plate interaction shifts the tip region of acoustic sensitivity clockwise or anti-clockwise about the trailing edge dependent upon the sign of the vortex. 


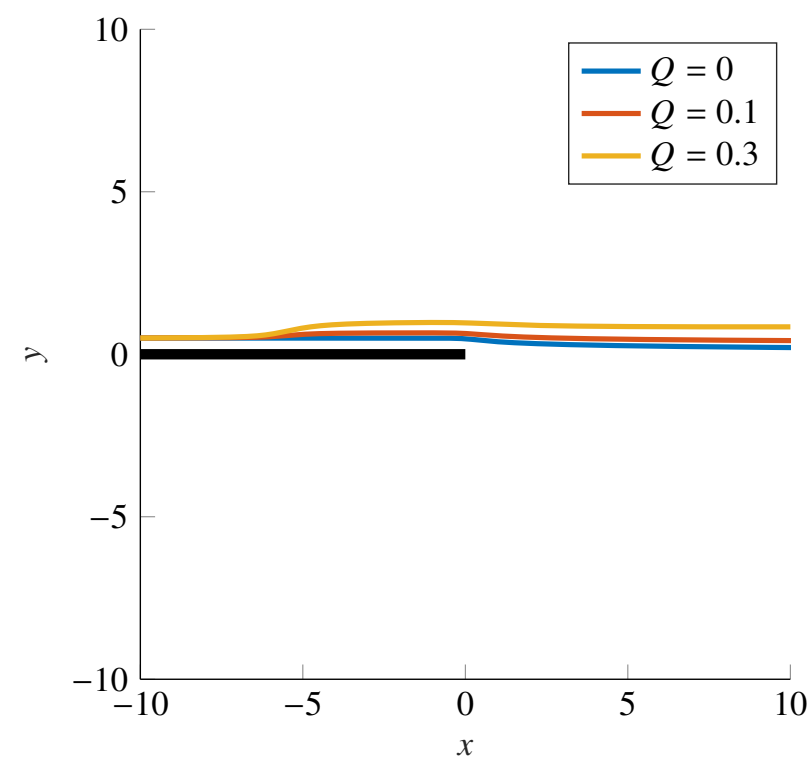

(a) Vortex paths

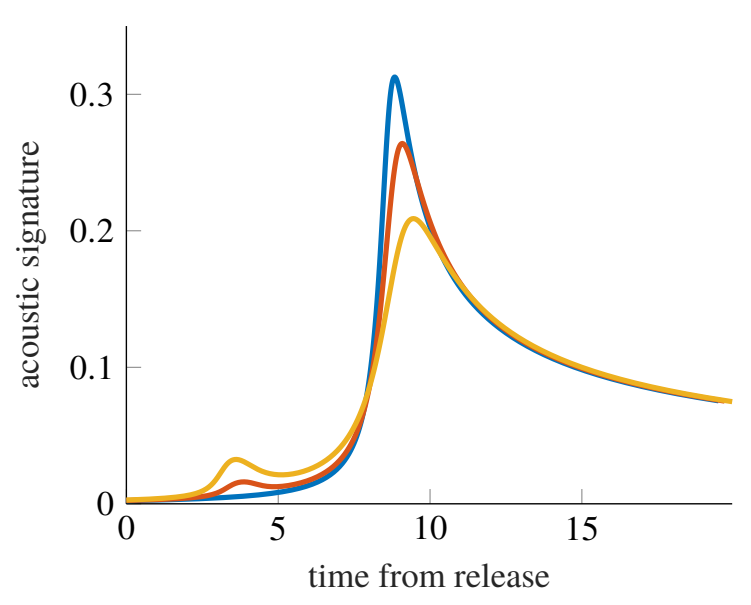

(b) Retarded far-field acoustic signature time history

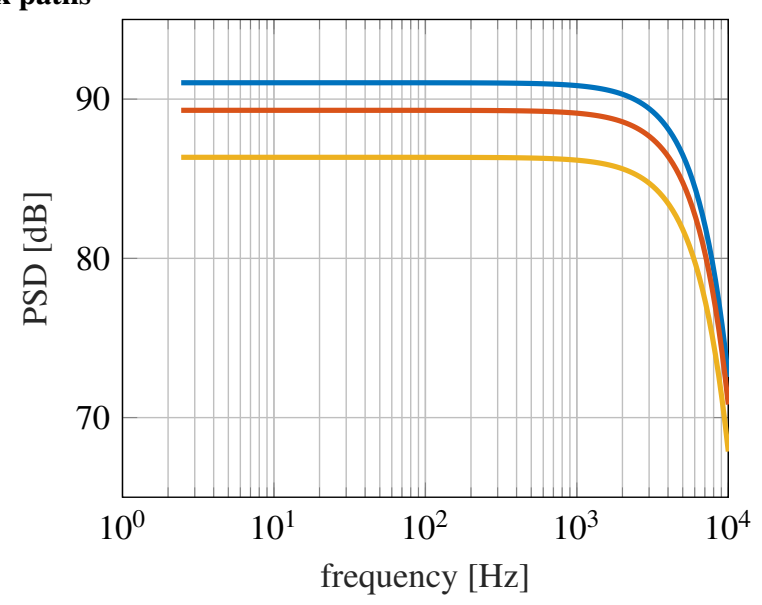

(c) Dimensionalised power spectral density [PSD] of the farfield acoustic signature

Fig. 16 Vortex sound results for a point vortex passing a trailing edge in active flow of different strengths at location $L=5$. The vortex is initialised at $-x_{\infty}+i y_{\infty}=-10+0.5 i$ and has strength $\Gamma=0.5$. 
reduction, this appears to be in line with the experimental results of [13] who report reductions in surface pressure fluctuations of up to $10-15 \mathrm{~dB}$ at higher frequencies, but additionally amplification of fluctuations at lower frequencies.

These results are robust to alternative blowing profiles of the same mass flux, with figure 14 indicating only small variations in the signature due to the active control interaction mainly resulting from the streamwise location of peak plate normal velocity. Larger deviations may be anticipated for vortices very close to the active device, and further work is required to understand strong interactions between the boundary layer, turbulent eddies and the active device. Such work is expected to elucidate the role of inclination on active flow performance.

\section{Acoustic signature decomposition}

The compact expression for the far-field acoustic signature (34) is linear in the $\zeta$-plane vortex velocity, which is a superposition of velocities induced by vortex-body, active and mean flow contributions. This enables identification of the respective instantaneous acoustic signature contributions. For anticipated realistic parameters the vortex self-interaction

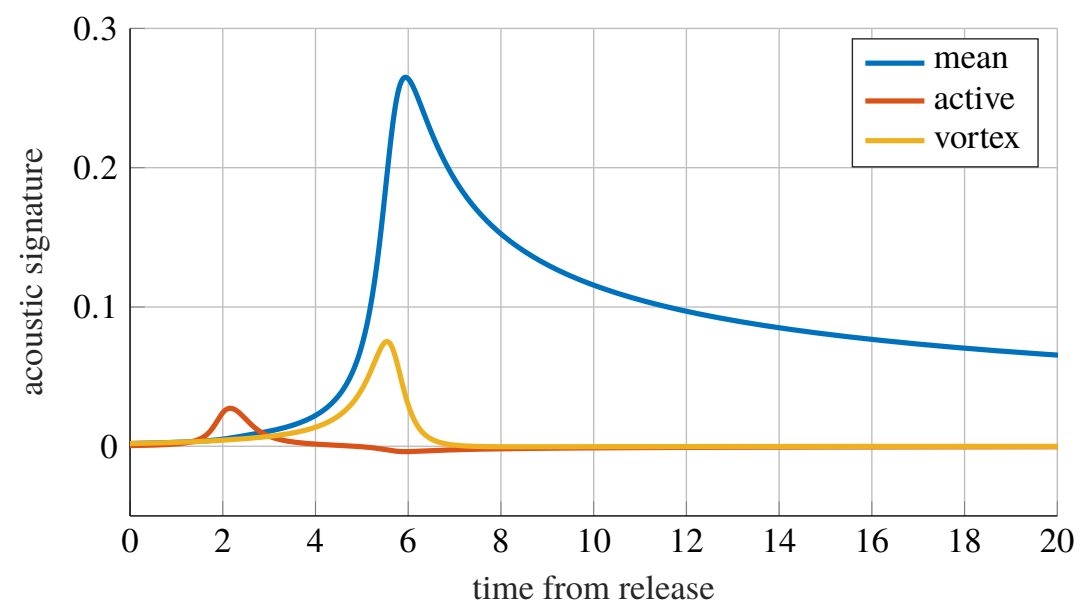

Fig. 17 Acoustic signature decomposition for active control at $L=5$. Active flow strength $Q=0.3$, vortex strength $\Gamma=0.5$, initialized at $-10+0.5 i$

and active flow terms are responsible for small local contributions to the acoustic signatures, but have a more appreciable effect upon acoustic signature through altering the global vortex path.

\section{Parameter optimization}

\section{Choice of objective function}

To quantify the radiated far-field sound we take the far-field compact acoustic signature (34) as a temporal density, and consider its $q$-norm (often referred to as a $p$-norm, we use $q$ to avoid confusion with pressure). This measure encompasses both a total acoustic energy radiated measure $(q=2)$ and the maximum absolute signature $(q=\infty)$. Large values of $q$ thus emphasise extreme values. The chosen norm may be expressed as

$$
\|P\|_{q}=\left(\int_{t_{1}}^{t_{2}}\left(\operatorname{Im}\left[\Gamma \frac{d \zeta_{0}}{d t}\right]\right)^{q} \mathrm{~d} t\right)^{1 / q}
$$

where we consider only a finite streamwise range about the trailing edge

$$
x_{0}\left(t_{1}\right)=x_{\min }, \quad x_{0}\left(t_{2}\right)=x_{\max }
$$

For simplicity we present results in figure $18 \mathrm{~b}$ on page 22 for cases $q=2,5$ and $\infty$, take $x_{\min }=-10, x_{\max }=10$, and consider the rapid distortion limit, taking a weaker vortex strength $\Gamma=0.1$. 


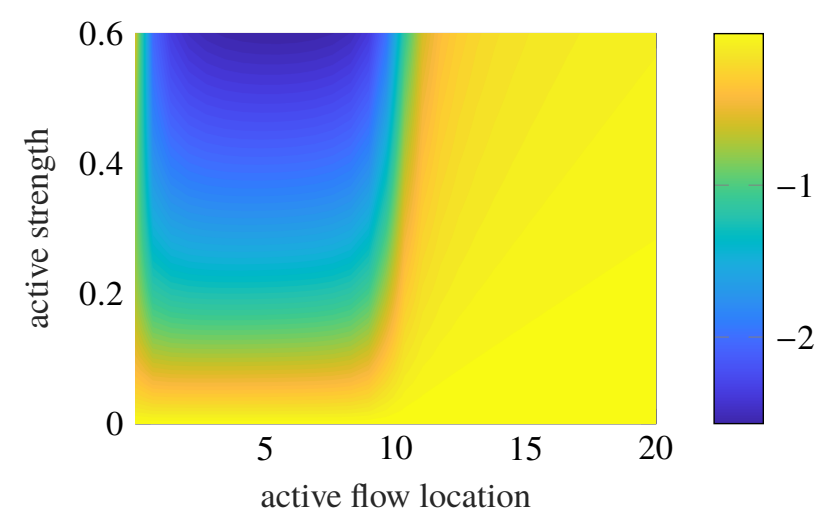

(a) initialized at $-10+0.5 i$

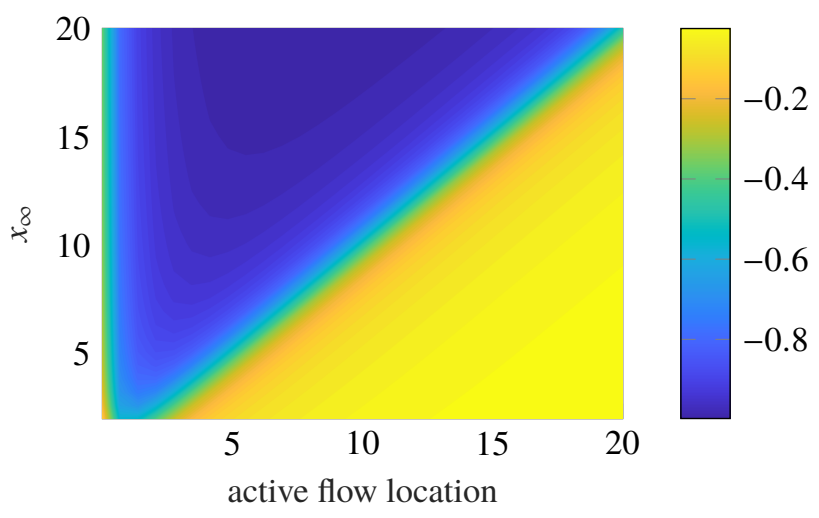

(b) active flow strength $Q=0.3$

Fig. 18 Comparison of reduction in peak far-field acoustic signature $[\mathrm{dB}]$ for vortex strength $\Gamma=0.5$, varying locations and strength.

\section{Optimal location of active control}

The optimal location is found to depend most strongly upon the streamwise initialization of the incident vortex. The far-field acoustic signature reduction for various active flow strengths, locations and streamwise vortex initialisations are presented in figure 18. For a single incident vortex in a weak blowing, results suggest the optimal placement of the control is approximately halfway between the vortex initialisation and the trailing edge. This result is largely robust to vortex strength and active strength. This optimal appears to be due to a maximal interaction between the control and the vortex, causing it to avoid the region of greatest acoustic sensitivity. To minimize the overall energy radiated as the vortex passes the trailing edge the optimal location of blowing moves further upstream, so as to keep the vortex further away from the edge for a longer time period. This must be balanced by a consideration of the development of the boundary layer over the aerofoil chord and downstream of the active control device which is not accounted for in the present model.

\section{Dependence on active control strength}

Figure 19 illustrates the dependence of acoustic signature reduction on active flow strength. Increasing the strength of the active flow, $Q$, causes the vortices to pass further from the edge, leading to a decreased far-field acoustic signature. This reduction becomes decreasingly significant as $Q$ is further increased. For sufficiently large active flow strengths and small normal displacements from the plate the vortex interaction with the active control becomes the dominant acoustic source. However, except for very small normal displacements this requires active flow strengths greater than the background mean flow $(Q>1)$. Negative values of $Q$, corresponding to suction, pull the vortex towards the trailing edge, leading to larger acoustic signatures if the vortex remains in the domain.

\section{Robustness to vortex model}

We now discuss the robustness of the model to vortex parameters to contextualise the results presented above. First, we note that there is a particularly sensitive dependence on the transverse displacement of the incident vortex from the plate, as may be anticipated by consideration of the sensitivity map (figure 15b) and is explicitly illustrated by figure 20a. Vorticity located closer to the trailing edge is seen to lead to substantial increases in acoustic emissions, and hence it becomes necessary to take into account the distribution of vorticity in the vicinity of the trailing edge. Moreover, it indicates the need to satisfactorily consider the role of vortex shedding [21].

Further, if we do not make the rapid distortion approximation, where an incident vortex travels along the streamlines of a background steady flow, more interesting dynamics arise. For vortices of moderate strengths near the plate, the vortex-body interaction has an appreciable effect upon the dynamics: a vortex is attracted to, or repulsed from, the trailing edge if the vortex has positive or negative sense. When a vortex of positive sense is sufficiently close to the plate, the vortex continues around the trailing edge, c.f. Crighton [20]. A vortex of negative sense sufficiently close to the edge will overcome the mean flow and move against the stream to $x=-\infty$, never reaching the trailing edge. This constrains the parameter values that we might expect to be valid for the phenomena considered, and further indicates 


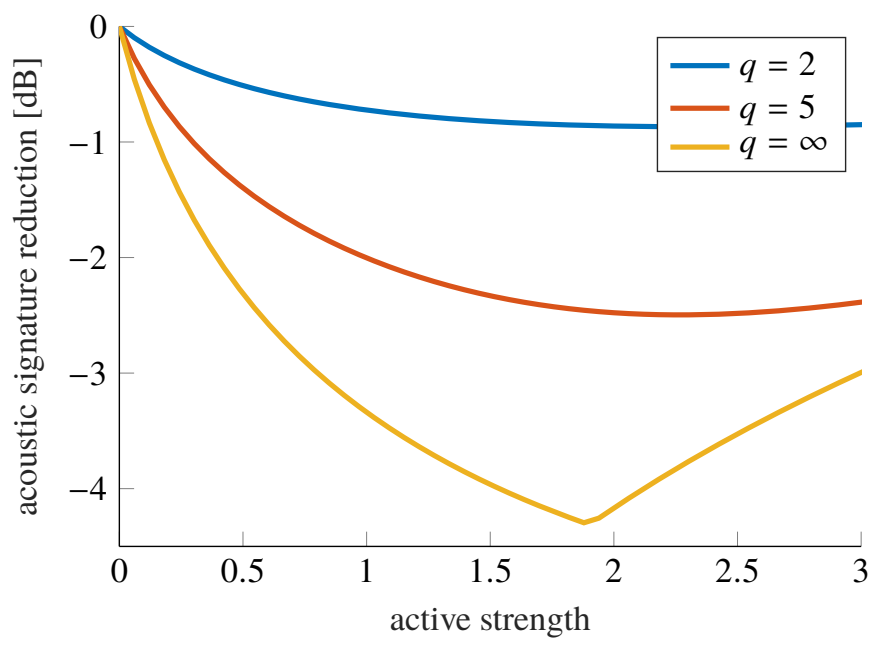

Fig. 19 Comparison of reduction in peak far-field acoustic signature for varying active strengths. We consider, in the RDT limit, a vortex of strength $\Gamma=0.1$ starting at displacement $-10+0.5 i$ in flow induced by active control at $L=5$.

the sensitivity of the model to the vortex parameters. Figure $20 \mathrm{~b}$ shows the predicted reduction in maximum far-field acoustic signature as the vortex strength and normal vortex displacement are varied, with the greyed regions indicating parameter values for which the vortex returns to $x=-\infty$.

Finally, our ability to infer robust realistic predictions from the above model is limited by our ability to translate between a singular eddy and a real flow disturbance. Considering a distributed set of singular disturbances may offer a more realistic picture of turbulent boundary layer trailing edge interaction and more natural translations from observed flows to the model, thus enabling fuller evaluation of the model.

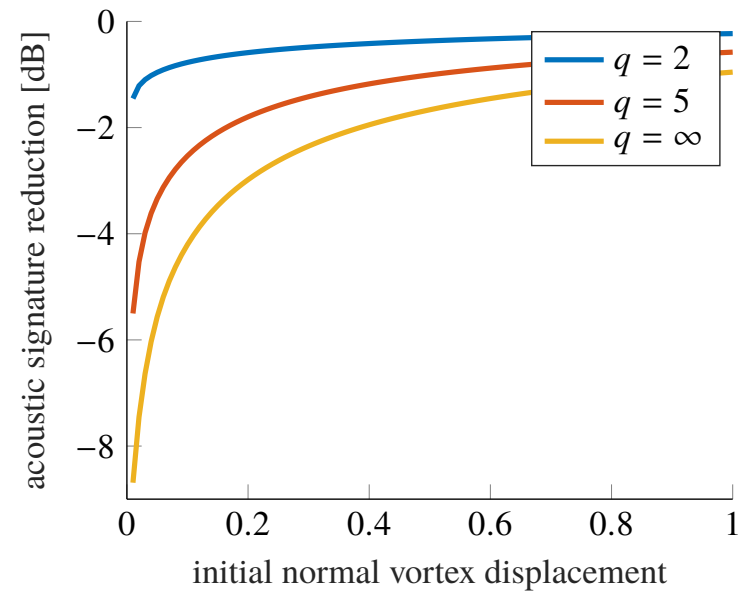

(a) RDT limit, vortex strength $\Gamma=0.1$

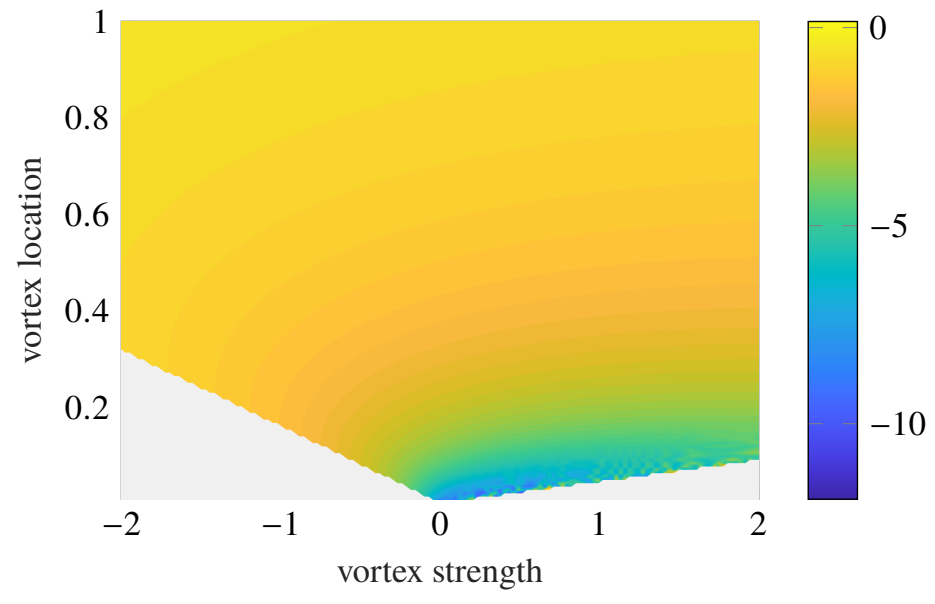

(b) Non-RDT limit

Fig. 20 Comparison of far-field acoustic signature reduction $P[\mathrm{~dB}]$ for various vortex parameters. We consider active control at $L=5$ with strength $Q=0.3$

\section{Conclusions}

Using a vortex sound framework, under a variety of assumptions about both active and the spanwise averaged nature of a three-dimensional flow, it is possible to investigate the noise-reduction properties of passive noise control devices, in particular finlets and other fence-like stuctures, aligned with the flow near a trailing-edge. Importantly, a 
noise reduction prediction can be obtained which depends only on the aspect ratio of the object encoded by $R$, and the fractional open area of the porous object, $\beta$. Whilst these results are difficult to generalise to geometries more complicated than elliptical objects near a flat trailing-edge, and they miss important physics important to the behaviour of such devices, a useful predictive tool is obtained with little additional complexity.

The vortex sound framework also provides an intuitive picture for gaining a theoretical understanding of active flow control. It indicates that active flow can offer appreciable reductions in trailing edge noise, suggesting a $2 \mathrm{~dB}$ reduction in peak acoustic signature may be reasonably anticipated. This is broadly consistent with previous experimental and numerical findings, considering the model's current constraints. Further work is required to understand the extent to which this model can offer insights into active flow phenomena, with natural developments to include general geometries, a full unsteady Kutta condition through vortex shedding [21] and background shear currently being pursued. Of particular interest is establishing the relative significance of vortical disturbances in different neighbourhoods of the trailing edge and better understanding the role of non-linear vortical interactions. Given the simplicity of the models considered, delineating effects which the vortex sound framework can realistically predict seems worthwhile in the hope of better understanding involved physical processes otherwise less approachable. Theoretical developments may help inform pursuits to implement active control effectively, and perhaps even efforts to alleviate practical obstacles to certain active flow methods, such as ingestion of particles by an active flow device.

\section{Acknowledgments}

M. J. Priddin thanks P. J. Baddoo for useful communications regarding the Plemelj formulae. L. J. Ayton acknowledges support from EPSRC Early Career Fellowship EP/P015980/1.

\section{References}

[1] Crighton, D. G., “Airframe noise,” Aeroacoustics of Flight Vehicles: Theory and Practice. Volume 1: Noise Sources, Vol. 1, 1991, pp. 391-447.

[2] Ffowcs Williams, J. E., and Hall, L. H., "Aerodynamic sound generation by turbulent flow in the vicinity of a scattering half plane," Journal of Fluid Mechanics, Vol. 40, No. 04, 1970, pp. 657-670.

[3] Lighthill, M. J., “On Sound Generated Aerodynamically. II. Turbulence as a Source of Sound,” Proceedings of the Royal Society A: Mathematical, Physical and Engineering Sciences, Vol. 222, No. 1148, 1954, pp. 1-32. doi:10.1098/rspa.1954.0049.

[4] Clark, I. A., Devenport, W. J., Jaworski, J. W., Daly, C., Peake, N., and Glegg, S., "The Noise Generating and Suppressing Characteristics of Bio-Inspired Rough Surfaces," AIAA/CEAS 20th Aeroacoustics Conference, Atlanta, GA, 2014.

[5] Clark, I., Baker, D., Alexander, W. N., Devenport, W., Peake, N., Glegg, S., and Jaworski, J., "Experimental and Theoretical Analysis of Bio-Inpsired Trailing Edge Noise Control Devices," AIAA/CEAS 22nd Aeroacoustics Conference, Lyon, 2016.

[6] Clark, I., Alexander, W. N., and Devenport, W., "Bio-Inspired Finlets for the Reduction of Marine Rotor Noise," 23rd AIAA/CEAS Aeroacoustics Conference, 2017, p. 3867.

[7] Afshari, A., Azarpeyvand, M., Dehghan, A. A., and Szóke, M., "Trailing Edge Noise Reduction Using Novel Surface Treatments," 22nd AIAA/CEAS Aeroacoustics Conference, AIAA, 2016, p. 2834. doi:10.2514/6.2016-2834.

[8] Afshari, A., Azarpeyvand, M., Dehghan, A. A., and Szóke, M., "Effects of Streamwise Surface Treatments on Trailing Edge Noise Reduction,” 2017. doi:10.2514/6.2017-3499.

[9] Bodling, A., Agrawal, B. R., Sharma, A., Clark, I., Alexander, W. N., and Devenport, W., "Numerical Investigation of Bioinspired Blade Designs at High Reynolds Numbers for Ultra-Quiet Aircraft and Wind Turbines," 2017. doi:10.2514/6.2017-3502.

[10] Jones, L. E., and Sandberg, R. D., "Acoustic and hydrodynamic analysis of the flow around an aerofoil with trailing-edge serrations,” J. Fluid Mech, Vol. 706, 2012, pp. 295-322. doi:10.1017/jfm.2012.254.

[11] Lyu, B., Azarpeyvand, M., and Sinayoko, S., "A Trailing-Edge Noise Model for Serrated Edges," 21 st AIAA/CEAS Aeroacoustics Conference, Vol. 2362, 2015. doi:10.2514/6.2015-2362.

[12] Gerhard, T., Erbslöh, S., and Carolus, T., "Reduction of airfoil trailing edge noise by trailing edge blowing," Journal of Physics: Conference Series, 2014. doi:10.1088/1742-6596/524/1/012123. 
[13] Szóke, M., and Azarpeyvand, M., "Active Flow Control Methods for the Reduction of Trailing Edge Noise," 23rd AIAA/CEAS Aeroacoustics Conference, 2017. doi:10.2514/6.2017-3004.

[14] Amiet, R. K., "Noise due to turbulent flow past a trailing edge," Journal of Sound and Vibration, 1976. doi:10.1016/0022460X(76)90948-2.

[15] Schlichting, H., Boundary-layer theory., $8^{\text {th }}$ ed., Springer-Verlag, Berlin, 2003.

[16] Cattafesta, L. N., and Sheplak, M., "Actuators for Active Flow Control," Annual Review of Fluid Mechanics, Vol. 43, No. 1, 2011, pp. 247-272. doi:10.1146/annurev-fluid-122109-160634.

[17] Semeraro, O., Bagheri, S., Brandt, L., and Henningson, D. S., "Transition delay in a boundary layer flow using active control," Journal of Fluid Mechanics, Vol. 731, 2013, p. 288-311. doi:10.1017/jfm.2013.299.

[18] Peake, N., and Crighton, D. G., "Active Control of Sound," Annual Review of Fluid Mechanics, Vol. 32, No. 1, 2000 , pp. 137-164. doi:10.1146/annurev.fluid.32.1.137.

[19] Reba, R. A., "A theoretical study of leading-edge devices for active and passive control of wake-airfoil interaction noise," Ph.D. thesis, Jan. 2000. 00001.

[20] Crighton, D. G., "Radiation from vortex filament motion near a half plane," Journal of Fluid Mechanics, Vol. 51, No. 02, 1972, pp. 357-362.

[21] Howe, M. S., “Trailing edge noise at low Mach numbers,” Journal of Sound and Vibration, Vol. 225, No. $2,1999$.

[22] Howe, M. S., Theory of Vortex Sound, Vol. 33, Cambridge University Press, 2003.

[23] Glegg, S. A. L., and Devenport, W., Aeroacoustics of low Mach number flows : fundamentals, analysis, and measurement, 2017.

[24] Jaworski, J., "Vortex sound generation from flexible fibers," 22nd AIAA/CEAS Aeroacoustics Conference, 2016, p. 2752.

[25] Clark, I. A., Daly, C. A., Devenport, W., Alexander, W. N., Peake, N., Jaworski, J. W., and Glegg, S., "Bio-inspired canopies for the reduction of roughness noise," Journal of Sound and Vibration, 2016. doi:10.1016/j.jsv.2016.08.027.

[26] Glegg, S., and Devenport, W. J., "Leading Edge Noise from Very Thick Airfoils with Vertical Fences," 23rd AIAA/CEAS Aeroacoustics Conference, 2017. doi:10.2514/6.2017-3495.

[27] Crighton, D. G., "The Kutta Condition in Unsteady Flow,” Ann. Rev. Fluid Mech, Vol. 17, 1985, pp. 411-45.

[28] Adams, M. C., "Leading-Edge Separation from Delta Wings at Supersonic Speeds," Journal of the Aeronautical Sciences, Vol. 20, No. 6, 1953, pp. 430-430. doi:10.2514/8.2663.

[29] Brown, C. E., and Michael, W. H., "Effect of Leading-Edge Separation on the Lift of a Delta Wing," Journal of the Aeronautical Sciences, Vol. 21, No. 10, 1954, pp. 690-694. doi:10.2514/8.3180.

[30] Smith, S. G. L., "How do singularities move in potential flow?" Physica D, Vol. 240, 2011, pp. 1644-1651. doi: 10.1016/j.physd.2011.06.010.

[31] Howe, M. S., "Emendation of the Brown \& Michael equation, with application to sound generation by vortex motion near a half-plane," Journal of Fluid Mechanics, Vol. 329, 1996, pp. 89-101.

[32] Howe, M. S., "Trailing edge noise at low Mach numbers, Part 2: Attached and separated edge flows," Journal of Sound and Vibration, Vol. 234, No. 5, 2000, pp. 761-775.

[33] Clark, I., Alexander, W. N., Devenport, W., Glegg, S., Jaworski, J., Daly, C., and Peake, N., "Bio-Inspired Trailing Edge Noise Control," North American Wind Energy Academy 2015 Symposium, 2015.

[34] Clark, I., Alexander, W. N., Devenport, W., Glegg, S., Jaworski, J., Daly, C., and Peake, N., "Bioinspired Trailing-Edge Noise Control," AIAA Journal, Vol. 55, No. 3, 2017, pp. 740-754. doi:10.2514/1.J055243.

[35] Graham, R. R., “The silent flight of owls,” Journal of the Royal Aeronautical Society, Vol. 38, 1934, pp. $837-843$.

[36] Geyer, T., Sarradj, E., and Fritzsche, C., "Measuring owl flight noise," INTER-NOISE and NOISE-CON Congress and Conference Proceedings, Institute of Noise Control Engineering, 2014, pp. 183-198.

[37] Kuethe, A. M., and Schetzer, J. D., Foundations of aerodynamics, Wiley, 1959. 
[38] Ablowitz, M. J., and Fokas, A. S., Complex variables: introduction and applications, Cambridge University Press, 2003.

[39] Driscoll, T. A., and Trefethen, L. N., Schwarz-Christoffel Mapping, Cambridge Monographs on Applied and Computational Mathematics, Cambridge University Press, 2002. doi:10.1017/CBO9780511546808. 